\title{
Correlation Coefficients of Interval Neutrosophic Hesitant Fuzzy Sets and Its Application in a Multiple Attribute Decision Making Method
}

\author{
Jun YE \\ Department of Electrical and Information Engineering, Shaoxing University, \\ 508 Huancheng West Road, Shaoxing, Zhejiang Province 312000, PR China \\ e-mail:yehjun@aliyun.com
}

Received: February 2014; accepted: January 2015

\begin{abstract}
This paper proposes the concept of an interval neutrosophic hesitant fuzzy set (INHFS) and the operational relations of INHFSs. Then, we develop correlation coefficients of INHFSs and investigate the relation between the similarity measures and the correlation coefficients. Furthermore, a multiple attribute decision making method based on the correlation coefficients is established under interval neutrosophic hesitant fuzzy environment. Through the correlation coefficients between each alternative and the ideal alternative, we obtain the ranking order of all alternatives and the best one. Finally, an illustrative example of investment alternatives is given to demonstrate the application and effectiveness of the developed approach.
\end{abstract}

Key words: interval neutrosophic hesitant fuzzy set, interval valued hesitant fuzzy set, interval neutrosophic set, correlation coefficient, multiple attribute decision making.

\section{Introduction}

Correlation coefficients are a powerful tool in data analysis and classification, decision making, pattern recognition and so on (Bonizzoni et al., 2008; Kriegel et al., 2008; Park et al., 2009; Szmidt and Kacprzyk, 2010; Ye, 2010; Wei et al., 2011). As many real world data may be fuzzy, various types of correlations have been proposed under fuzzy environments. Chiang and Lin (1999) introduced the concept of correlation. Hong (2006) proposed a fuzzy correlation coefficient under Tw (the weakest t-norm) based fuzzy arithmetic operations. As an extension of fuzzy correlations, Wang and Li (1999) introduced the correlation and information energy of interval-valued fuzzy numbers. Then, Gerstenkorn and Manko (1991) developed the correlation coefficients of intuitionistic fuzzy sets (IFSs). Also, Hung and Wu (2002) proposed a method to calculate the correlation coefficients of IFSs by means of "centroid". Furthermore, Bustince and Burillo (1995) and Hong (1998) further developed the correlation coefficients for interval-valued intuitionistic fuzzy sets (IVIFSs). Recently, Torra and Narukawa (2009) and Torra (2010) extended fuzzy sets to hesitant fuzzy sets (HFSs), which allow the membership degree of an element to a set represented by several possible values. Thus, HFS can be considered as a powerful tool to express uncertain information in the process of group decision 
making. After that, Chen et al. (2013a) proposed the concept of interval valued hesitant fuzzy sets (IVHFSs) and some correlation coefficients for HFSs and IVHFSs and applied them to clustering analysis under hesitant fuzzy environments and interval hesitant fuzzy environments, and then they (Chen et al., 2013b) further proposed interval valued hesitant preference relations and applied them to group decision making. As a further generalization of the correlation coefficient between HFSs, Ye (2014a) presented the correlation coefficient of dual hesitant fuzzy sets (DHFSs) and applied it to multiple attribute decision making problems with duel hesitant fuzzy information. Recently, intuitionistic (uncertain) linguistic numbers and their applications in decision making have received more and more attention (Liu and Jin, 2012; Liu, 2013; Liu and Wang, 2014; Liu et al., 2014).

However, the aforementioned sets can only handle incomplete information but not the indeterminate information and inconsistent information which exist commonly in real situations. For example, for a given proposition "movie $X$ would be a hit", in this situation human brain certainly cannot generate precise answers in terms of yes or no, as indeterminacy is the sector of unawareness of a proposition's value between truth and falsity. Thus, Smarandache (1999) presented a neutrosophic set from philosophical point of view. The neutrosophic set is a powerful general formal framework which generalizes the concept of the classic set, fuzzy set, interval-valued fuzzy set (IVFS), IFS, IVIFS, paraconsistent set, dialetheist set, paradoxist set, and tautological set (Smarandache, 1999). In the neutrosophic set, indeterminacy is quantified explicitly and truth-membership, indeterminacymembership, and falsity-membership are independent. Its functions $T_{A}(x), I_{A}(x)$ and $F_{A}(x)$ are real standard or nonstandard subsets of $]^{-} 0,1^{+}$[, i.e., $\left.T_{A}(x): X \rightarrow\right]^{-} 0,1^{+}[$, $\left.I_{A}(x): X \rightarrow\right]^{-} 0,1^{+}\left[\text {, and } F_{A}(x): X \rightarrow\right]^{-} 0,1^{+}$[. Obviously, the neutrosophic components are best fit in the representation of indeterminacy and inconsistent information. However, it will be difficult to apply in real scientific and engineering areas. Therefore, Wang et al. $(2005,2010)$ proposed the concepts of an interval neutrosophic set (INS) and a single valued neutrosophic set (SVNS), which are the subclasses of a neutrosophic set, and provided the set-theoretic operators and various properties of SVNSs and INSs. Thus, SVNSs and INSs can be applied in real scientific and engineering fields and give us an additional possibility to represent uncertainty, imprecise, incomplete, and inconsistent information which exists in real world. Recently, Ye (2013) presented the correlation coefficient of SVNSs based on the extension of the correlation coefficient of intuitionistic fuzzy sets and proved that the cosine similarity measure of SVNSs is a special case of the correlation coefficient of SVNSs, and then applied it to single valued neutrosophic decision-making problems. Then, Broumi and Smarandache (2013) introduced the correlation coefficient of INSs. On the other hand, Ye (2014b) developed a single valued neutrosophic crossentropy measure and applied it to decision-making problems with single valued neutrosophic information. Ye (2014c) also introduced the Hamming and Euclidean distances between INSs and their similarity measures, and then applied them to decision-making problems with interval neutrosophic information. Furthermore, Ye (2014d) presented a concept of a simplified neutrosophic set (SNS), which is a subclass of the neutrosophic set and includes a SVNS and an INS, and defined some operations of SNSs, and then he 
developed a simplified neutrosophic weighted averaging (SNWA) operator, a simplified neutrosophic weighted geometric (SNWG) operator, and a multicriteria decision-making method based on the SNWA and SNWG operators and the cosine measure of SNSs under simplified neutrosophic environment.

The IVHFS, which allows the membership degree of an element to a set represented by several possible interval values, can be considered as a powerful tool to express uncertain information in the group decision-making process. But it cannot handle indeterminate and inconsistent information, while the INS gives us an additional possibility to represent uncertainty, imprecise, incomplete, and inconsistent information which exists in real world and would be more suitable to handle indeterminate information and inconsistent information. However, existing SVNSs and INSs cannot allow truth-membership degrees, indeterminacy-membership degrees and falsity-membership degrees of an element to a set represented by several possible values or interval values, and then existing correlation coefficients for SVNSs and INSs cannot also handle this hesitant problem. To solve this problem, it is very necessary to introduce the concept of interval neutrosophic hesitant fuzzy sets (INHFSs), which permit truth-membership degrees, indeterminacymembership degrees, and falsity-membership degrees of an element to a given set to have a few different interval values. Hence, the INHFS encompasses fuzzy set, IFS, IVIFS, SVNS, INS, HFS, DHFS, and IVHFS as special cases of the INHFS. The purposes of this paper are: (1) to propose the concept of INHFSs based on the combination of INSs and IVHFSs and some basic operations of INHFSs, (2) to develop some correlation coefficients between INHFSs and to investigate their properties and the relation between the correlation coefficients and some similarity measures, and (3) to establish a multiple attribute decision making method based on the correlation coefficients under interval neutrosophic hesitant fuzzy environment. The proposed decision making method based on the correlation coefficients can avoid complex information aggregation and can directly utilize the derived correlation coefficients to calculate the correlation degrees between alternatives and the ideal alternative for the ranking order of the alternatives.

The rest of the paper is organized as follows. Section 2 briefly describes some concepts of INSs, HFSs, IVHFSs, and the correlation coefficients between IVHFSs. Section 3 proposes the concept of INHFSs and defines the corresponding basic operations. In Section 4, we develop some correlation coefficients between INHFSs and investigate their properties and the relation between some similarity measures and the correlation coefficients. Section 5 establishes a decision-making approach based on the correlation coefficients under interval neutrosophic hesitant fuzzy environment. An illustrative example validating our approach is presented in Section 6. Section 7 gives conclusions and some remarks.

\section{Preliminaries}

\subsection{Interval Neutrosophic Set}

Smarandache (1999) presented the neutrosophic set from philosophical point of view and gave the following definition of a neutrosophic set. 


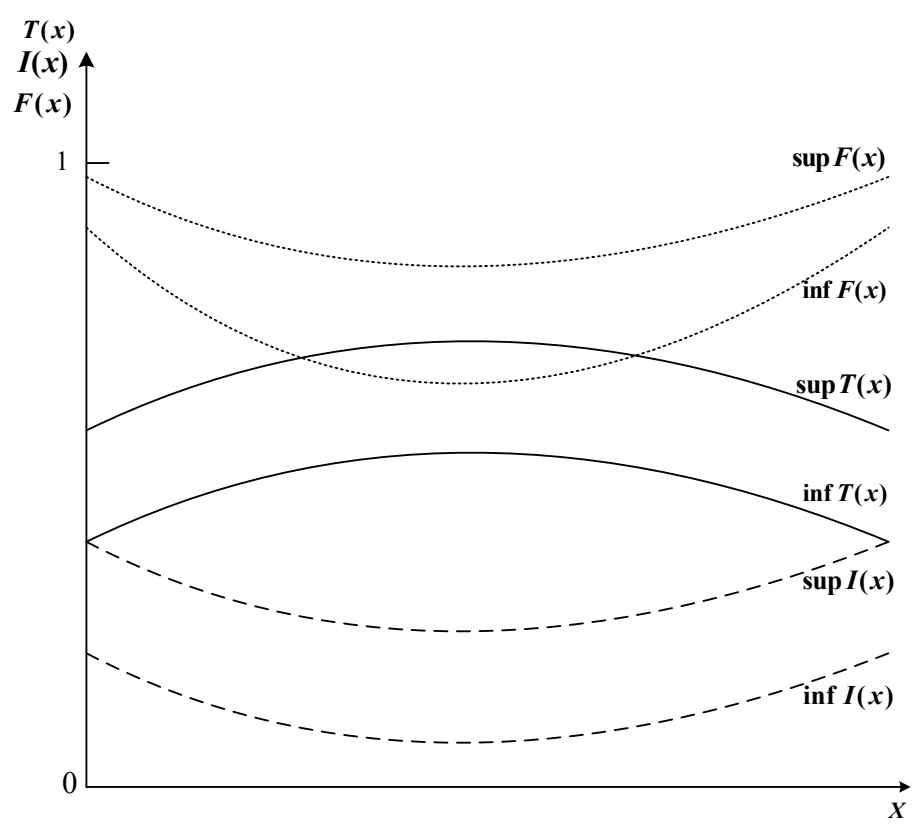

Fig. 1. Illustration of an INS in $R^{1}$.

Definition 1. (See Smarandache, 1999.) Let $X$ be a space of points (objects), with a generic element in $X$ denoted by $x$. A neutrosophic set $A$ in $X$ is characterized by a truthmembership function $T_{A}(x)$, an indeterminacy-membership function $I_{A}(x)$, and a falsitymembership function $F_{A}(x)$. The functions $T_{A}(x), I_{A}(x)$ and $F_{A}(x)$ are real standard or nonstandard subsets of $]^{-} 0,1^{+}$[, i.e., $\left.T_{A}(x): X \rightarrow\right]^{-} 0,1^{+}\left[, I_{A}(x): X \rightarrow\right]^{-} 0,1^{+}[$, and $\left.F_{A}(x): X \rightarrow\right]^{-} 0,1^{+}\left[\right.$. There is no restriction on the sum of $T_{A}(x), I_{A}(x)$ and $F_{A}(x)$, so $-0 \leqslant \sup T_{A}(x)+\sup I_{A}(x)+\sup F_{A}(x) \leqslant 3^{+}$.

Obviously, it is difficult to apply the neutrosophic set to practical problems. Therefore, Wang et al. (2005) introduced the concept of an INS, which is a subclass of the neutrosophic set, for real scientific and engineering applications. In the following, we introduce the definition of an INS (Wang et al., 2005).

Definition 2. (See Wang et al., 2005.) Let $X$ be a space of points (objects) with generic elements in $X$ denoted by $x$. An INS $A$ in $X$ is characterized by a truth-membership function $T_{A}(x)$, an indeterminacy-membership function $I_{A}(x)$, and a falsity-membership function $F_{A}(x)$, where $T_{A}(x), I_{A}(x), F_{A}(x) \subseteq[0,1]$ for each point $x$ in $X$. Hence, the sum of $T_{A}(x), I_{A}(x)$ and $F_{A}(x)$ is $0 \leqslant \sup T_{A}(x)+\sup I_{A}(x)+\sup F_{A}(x) \leqslant 3$.

We call it "interval" because it is the subclass of a neutrosophic set, that is, we only consider the subunitary interval of $[0,1]$. Therefore, all INSs are clearly neutrosophic sets. An INS in $R^{1}$ is illustrated in Fig. 1 (Wang et al., 2005).

Definition 3. (See Wang et al., 2005.) An INS $A$ is empty if and only if its $\inf T_{A}(x)=$ $\sup T_{A}(x)=0, \inf I_{A}(x)=\sup I_{A}(x)=1$, and inf $F_{A}(x)=\sup F_{A}(x)=0$ for any $x$ in $X$. 
Definition 4. (See Wang et al., 2005.) The complement of an INS $A$ is denoted by $A^{c}$ and is defined as $T_{A}^{c}(x)=F_{A}(x), \inf I_{A}^{c}(x)=1-\sup I_{A}(x), \sup I_{A}^{c}(x)=1-\inf I_{A}(x)$, $F_{A}^{c}(x)=T_{A}(x)$ for any $x$ in $X$.

Definition 5. (See Wang et al., 2005.) An INS $A$ is contained in the other INS $B, A \subseteq B$, if and only if $\inf T_{A}(x) \leqslant \inf T_{B}(x), \sup T_{A}(x) \leqslant \sup T_{B}(x), \inf I_{A}(x) \geqslant \inf I_{B}(x)$, $\sup I_{A}(x) \geqslant \sup I_{B}(x), \inf F_{A}(x) \geqslant \inf F_{B}(x)$, and $\sup F_{A}(x) \geqslant \sup F_{B}(x)$ for any $x$ in $X$.

Definition 6. (See Wang et al., 2005.) Two INSs $A$ and $B$ are equal, written as $A=B$, if and only if $A \subseteq B$ and $B \subseteq A$.

\subsection{Some Basic Concepts of HFSs and IVHFSs}

Torra and Narukawa (2009) and Torra (2010) firstly proposed the concept of a HFS, which is defined as follows:

Definition 7. (See Torra and Narukawa, 2009; Torra, 2010.) Let $X$ be a fixed set, a HFS $A$ on $X$ is defined in terms of a function $h_{A}(x)$ that when applied to $X$ returns a finite subset of $[0,1]$, which can be represented as the following mathematical symbol:

$$
A=\left\{\left\langle x, h_{A}(x)\right\rangle \mid x \in X\right\}
$$

where $h_{A}(x)$ is a set of some different values in $[0,1]$, denoting the possible membership degrees of the element $x \in X$ to $A$. For convenience, we call $h_{A}(x)$ a hesitant fuzzy element, denoted by $h$.

Given three hesitant fuzzy elements $h, h_{1}$, and $h_{2}$, Torra (2010) defined some operations in them as follows:

(1) $h^{c}=\bigcup_{\gamma \in h}\{1-\gamma\}$,

(2) $h_{1} \cup h_{2}=\bigcup_{\gamma_{1} \in h_{1}, \gamma_{2} \in h_{2}} \max \left\{\gamma_{1}, \gamma_{2}\right\}$,

(3) $h_{1} \cap h_{2}=\bigcup_{\gamma_{1} \in h_{1}, \gamma_{2} \in h_{2}} \min \left\{\gamma_{1}, \gamma_{2}\right\}$.

Then, Xia and Xu (2011) defined some operations on the hesitant fuzzy elements $h$, $h_{1}, h_{2}$ and a positive scale $\lambda$ :

(1) $h^{\lambda}=\bigcup_{\gamma \in h}\left\{\gamma^{\lambda}\right\}$,

(2) $\lambda h=\bigcup_{\gamma \in h}\left\{1-(1-\gamma)^{\lambda}\right\}$,

(3) $h_{1} \oplus h_{2}=\bigcup_{\gamma_{1} \in h_{1}, \gamma_{2} \in h_{2}}\left\{\gamma_{1}+\gamma_{2}-\gamma_{1} \gamma_{2}\right\}$,

(4) $h_{1} \otimes h_{2}=\bigcup_{\gamma_{1} \in h_{1}, \gamma_{2} \in h_{2}}\left\{\gamma_{1} \gamma_{2}\right\}$.

Definition 8. (See Chen et al., 2013a, 2013b.) Let $X$ be a fixed set, an IVHFS on $X$ is defined as:

$$
E=\left\{\left\langle x, \tilde{h}_{E}(x)\right\rangle \mid x \in X\right\}
$$


where $\tilde{h}_{E}(x)$ is a set of some different interval values in $[0,1]$, representing the possible membership degrees of the element $x \in X$ to the set $E$, and is called an interval valued hesitant fuzzy element (IVHFE). For convenience, $\tilde{h}_{E}(x)$ is denoted by an IVHFE $\tilde{h}$, which reads $\tilde{h}=\{\tilde{\gamma} \mid \tilde{\gamma} \in \tilde{h}\}$, where $\tilde{\gamma}=\left[\gamma^{L}, \gamma^{U}\right]$ is an interval number, $\gamma^{L}=\inf \tilde{\gamma}$ and $\gamma^{U}=\sup \tilde{\gamma}$ represent the lower and upper limits of $\tilde{\gamma}$, respectively.

Given three IVHFEs $\tilde{h}, \tilde{h}_{1}, \tilde{h}_{2}$, and a positive scale $\lambda>0$, their operations (Chen $e t$ al., 2013b) can be defined as follows:

(1) $\tilde{h}^{\lambda}=\bigcup_{\tilde{\gamma} \in \tilde{h}}\left\{\left[\left(\gamma^{L}\right)^{\lambda},\left(\gamma^{U}\right)^{\lambda}\right]\right\}$;

(2) $\lambda \tilde{h}=\bigcup_{\tilde{\gamma} \in \tilde{h}}\left\{\left[1-\left(1-\gamma^{L}\right)^{\lambda}, 1-\left(1-\gamma^{U}\right)^{\lambda}\right]\right\}$;

(3) $\tilde{h}_{1} \oplus \tilde{h}_{2}=\bigcup_{\tilde{\gamma}_{1} \in \tilde{h}_{1}, \tilde{\gamma}_{2} \in \tilde{h}_{2}}\left\{\left[\gamma_{1}^{L}+\gamma_{2}^{L}-\gamma_{1}^{L} \gamma_{2}^{U}, \gamma_{1}^{U}+\gamma_{2}^{U}-\gamma_{1}^{U} \gamma_{2}^{U}\right]\right\}$;

(4) $\tilde{h}_{1} \otimes \tilde{h}_{2}=\bigcup_{\tilde{\gamma}_{1} \in \tilde{h}_{1}, \tilde{\gamma}_{2} \in \tilde{h}_{2}}\left\{\left[\gamma_{1}^{L} \gamma_{2}^{L}, \gamma_{1}^{U} \gamma_{2}^{U}\right]\right\}$.

To compare two interval numbers, we introduce the following definition (Chen et al., 2013b).

Definition 9. (See Chen et al., 2013b.) Let $\tilde{a}=\left[a^{L}, a^{U}\right]$ and $\tilde{b}=\left[b^{L}, b^{U}\right]$ be two interval numbers and $l_{\tilde{a}}=a^{U}-a^{L}$ and $l_{\tilde{b}}=b^{U}-b^{L}$, then the degree of possibility of $\tilde{a} \geqslant \tilde{b}$ is formulated by

$$
p(\tilde{a} \geqslant \tilde{b})=\max \left[1-\max \left(\frac{b^{U}-a^{L}}{l_{\tilde{a}}+l_{\tilde{b}}}, 0\right), 0\right] .
$$

\subsection{Correlation Coefficients of IVHFSs}

Since the number of interval elements in different IVHFEs could be different and the interval numbers are usually disorder, we can arrange them in an increasing order using Eq. (1) for the comparison between two interval numbers. For any IVHFE $\tilde{h}=\{\tilde{\gamma} \mid \tilde{\gamma} \in \tilde{h}\}$, let $\tilde{\gamma}_{\sigma(j)}$ stand for the $j$ th smallest value in $\tilde{h}$, where $(\sigma(1), \sigma(2), \ldots, \sigma(n))$ is a permutation of $(1,2, \ldots, n)$, such that $\tilde{\gamma}_{\sigma(j)} \leqslant \tilde{\gamma}_{\sigma(j+1)}$ for $j=1,2, \ldots, n-1$. For two IVHFSs $A=$ $\left\{\left\langle x_{i}, \tilde{h}_{A}\left(x_{i}\right)\right\rangle \mid x_{i} \in X, i=1,2, \ldots, n\right\}$ and $B=\left\{\left\langle x_{i}, \tilde{h}_{B}\left(x_{i}\right)\right\rangle \mid x_{i} \in X, i=1,2, \ldots, n\right\}$, to calculate the correlation coefficient between $A$ and $B$, let $l_{i}=\max \left[l\left(\tilde{h}_{A}\left(x_{i}\right)\right), l\left(\tilde{h}_{B}\left(x_{i}\right)\right)\right]$ for each $x_{i}$ in $X$, where $l\left(\tilde{h}_{A}\left(x_{i}\right)\right)$ and $l\left(\tilde{h}_{B}\left(x_{i}\right)\right)$ represent the number of interval elements (interval numbers) in $\tilde{h}_{A}\left(x_{i}\right)$ and $\tilde{h}_{B}\left(x_{i}\right)$, respectively. If $l\left(\tilde{h}_{A}\left(x_{i}\right)\right) \neq l\left(\tilde{h}_{B}\left(x_{i}\right)\right)$, according to the optimistic principle, one can add the maximum value in less number of elements between $\tilde{h}_{A}\left(x_{i}\right)$ and $\tilde{h}_{B}\left(x_{i}\right)$ until them have the same length between $\tilde{h}_{A}\left(x_{i}\right)$ and $\tilde{h}_{B}\left(x_{i}\right)$. Thus, Chen et al. (2013a) gave two correlation coefficients of the IVHFSs $A$ and $B$ as follows:

$$
\begin{aligned}
R_{I V H F S I}(A, B)= & \frac{C_{I V H F S}(A, B)}{\left[C_{I V H F S}(A, A) \cdot C_{I V H F S}(B, B)\right]^{1 / 2}} \\
= & \frac{\sum_{i=1}^{n}\left(\frac{1}{l_{i}} \sum_{j=1}^{l_{i}}\left(\gamma_{A i \sigma(j)}^{L} \gamma_{B i \sigma(j)}^{L}+\gamma_{A i \sigma(j)}^{U} \gamma_{B i \sigma(j)}^{U}\right)\right)}{\left\{\sum_{i=1}^{n}\left\{\frac{1}{l_{i}} \sum_{j=1}^{l_{i}}\left[\left(\gamma_{A i \sigma(j)}^{L}\right)^{2}+\left(\gamma_{A i \sigma(j)}^{U}\right)^{2}\right]\right\}\right\}^{1 / 2}} \\
& \quad \times\left\{\sum_{i=1}^{n}\left\{\frac{1}{l_{i}} \sum_{j=1}^{l_{i}}\left[\left(\gamma_{B i \sigma(j)}^{L}\right)^{2}+\left(\gamma_{B i \sigma(j)}^{U}\right)^{2}\right]\right\}\right\}^{1 / 2}
\end{aligned}
$$




$$
\begin{aligned}
& R_{I V H F S 2}(A, B)=\frac{C_{I V H F S}(A, B)}{\max \left[C_{I V H F S}(A, A), C_{I V H F S}(B, B)\right]} \\
& =\frac{\sum_{i=1}^{n}\left(\frac{1}{l_{i}} \sum_{j=1}^{l_{i}}\left(\gamma_{A i \sigma(j)}^{L} \gamma_{B i \sigma(j)}^{L}+\gamma_{A i \sigma(j)}^{U} \gamma_{B i \sigma(j)}^{U}\right)\right)}{\max \left\{\sum_{i=1}^{n}\left\{\frac{1}{l_{i}} \sum_{j=1}^{l_{i}}\left[\left(\gamma_{A i \sigma(j)}^{L}\right)^{2}+\left(\gamma_{A i \sigma(j)}^{U}\right)^{2}\right]\right\},\right.} \\
& \left.\sum_{i=1}^{n}\left\{\frac{1}{l_{i}} \sum_{j=1}^{l_{i}}\left[\left(\gamma_{B i \sigma(j)}^{L}\right)^{2}+\left(\gamma_{B i \sigma(j)}^{U}\right)^{2}\right]\right\}\right\}
\end{aligned}
$$

where $\tilde{\gamma}_{A i \sigma(j)}=\left[\gamma_{A i \sigma(j)}^{L}, \gamma_{A i \sigma(j)}^{U}\right] \in \tilde{h}_{A}\left(x_{i}\right)$ and $\tilde{\gamma}_{B i \sigma(j)}=\left[\gamma_{B i \sigma(j)}^{L}, \gamma_{B i \sigma(j)}^{U}\right] \in \tilde{h}_{B}\left(x_{i}\right)$ for $i=1,2, \ldots, n$ and $j=1,2, \ldots, l_{i}$.

The two correlation coefficients of $R_{\text {IVHFSk }}(A, B)(k=1,2)$ satisfy the following properties (Chen et al., 2013a):

(1) $R_{\text {IVHFSk }}(A, B)=R_{I V H F S k}(B, A)$;

(2) $0 \leqslant R_{I V H F S k}(A, B) \leqslant 1$;

(3) $R_{\text {IVHFSk }}(A, B)=1$, if $A=B$.

\section{Interval Neutrosophic Hesitant Fuzzy Set}

In this section, the concept of an INHFS is presented based on the combination of INSs and IVHFSs as a further generalization of that of INSs and IVHFSs, which is defined as follows.

Definition 10. Let $X$ be a fixed set, an INHFS on $X$ is defined as

$$
N=\{\langle x, \tilde{t}(x), \tilde{i}(x), \tilde{f}(x)\rangle \mid x \in X\}
$$

where $\tilde{t}(x), \tilde{i}(x)$, and $\tilde{f}(x)$ are sets of some different interval values in $[0,1]$, representing the possible truth-membership hesitant degrees, indeterminacy-membership hesitant degrees, and falsity-membership hesitant degrees of the element $x \in X$ to the set $N$, respectively. Then, $\tilde{t}(x)$ reads $\tilde{t}(x)=\{\tilde{\gamma} \mid \tilde{\gamma} \in \tilde{t}(x)\}$, where $\tilde{\gamma}=\left[\gamma^{L}, \gamma^{U}\right]$ is an interval number, $\gamma^{L}=\inf \tilde{\gamma}$ and $\gamma^{U}=\sup \tilde{\gamma}$ represent the lower and upper limits of $\tilde{\gamma}$, respectively; $\tilde{i}(x)$ reads $\tilde{i}(x)=\{\tilde{\delta} \mid \tilde{\delta} \in \tilde{i}(x)\}$, where $\tilde{\delta}=\left[\delta^{L}, \delta^{U}\right]$ is an interval number, $\delta^{L}=\inf \tilde{\delta}$ and $\delta^{U}=\sup \tilde{\delta}$ represent the lower and upper limits of $\tilde{\delta}$, respectively; $\tilde{f}(x)$ reads $\tilde{f}(x)=$ $\{\tilde{\eta} \mid \tilde{\eta} \in \tilde{f}(x)\}$, where $\tilde{\eta}=\left[\eta^{L}, \eta^{U}\right]$ is an interval number, $\eta^{L}=\inf \tilde{\eta}$ and $\eta^{U}=\sup \tilde{\eta}$ represent the lower and upper limits of $\tilde{\eta}$, respectively. Hence, there is the condition $0 \leqslant \sup \gamma^{+}+\sup \delta^{+}+\sup \eta^{+} \leqslant 3$, where $\tilde{\gamma}^{+}=\bigcup_{\tilde{\gamma} \in \tilde{t}(x)} \max \{\tilde{\gamma}\}, \tilde{\delta}^{+}=\bigcup_{\tilde{\delta} \in \tilde{i}(x)} \max \{\tilde{\delta}\}$, and $\tilde{\eta}^{+}=\bigcup_{\tilde{\eta} \in \tilde{f}(x)} \max \{\tilde{\eta}\}$ for $x \in X$.

For convenience, the three tuple $\tilde{n}(x)=\{\tilde{t}(x), \tilde{i}(x), \tilde{f}(x)\}$ is called an interval neutrosophic hesitant fuzzy element (INHFE), which is denoted by the simplified symbol $\tilde{n}=\{\tilde{t}, \tilde{i}, \tilde{f}\}$.

From Definition 10, it is obvious that the INHFS consists of three parts of the truthmembership, the indeterminacy-membership, and the falsity-membership, supporting 
a more exemplary and flexible access to assign some interval values for each element in the domain, and can handle three kinds of hesitancy in this situation. Thus, the existing sets, including fuzzy sets, IFSs, IVIFSs, SVNSs, INSs, HFSs, DHFSs, and IVHFSs, can be regarded as special cases of INHFSs.

Then, we can define the union and intersection of INHFEs as the follows:

Definition 11. Let $\tilde{n}_{1}=\left\{\tilde{t}_{1}, \tilde{i}_{1}, \tilde{f}_{1}\right\}$ and $\tilde{n}_{2}=\left\{\tilde{t}_{2}, \tilde{i}_{2}, \tilde{f}_{2}\right\}$ be two INHFEs in a fixed set $X$, then their union and intersection are defined, respectively, by

(1) $\tilde{n}_{1} \cup \tilde{n}_{2}=\left\{\tilde{t}_{1} \cup \tilde{t}_{2}, \tilde{i}_{1} \cap \tilde{i}_{2}, \tilde{f}_{1} \cap \tilde{f}_{2}\right\}$,

(2) $\tilde{n}_{1} \cap \tilde{n}_{2}=\left\{\tilde{t}_{1} \cap \tilde{t}_{2}, \tilde{i}_{1} \cup \tilde{i}_{2}, \tilde{f}_{1} \cup \tilde{f}_{2}\right\}$.

Hence, for two INHFEs $\tilde{n}_{1}, \tilde{n}_{2}$, and a positive scale $\lambda>0$, the basic operations can be given as follows:

(1) $\tilde{n}_{1} \oplus \tilde{n}_{2}=\left\{\tilde{t}_{1} \oplus \tilde{t}_{2}, \tilde{i}_{1} \otimes \tilde{i}_{2}, \tilde{f}_{1} \otimes \tilde{f}_{2}\right\}$

$$
\begin{aligned}
= & \cup \\
& \tilde{\gamma}_{1} \in \tilde{t}_{1}, \tilde{\delta}_{1} \in \tilde{i}_{1}, \tilde{\eta}_{1} \in \tilde{f}_{1}, \tilde{\gamma}_{2} \in \tilde{t}_{2}, \tilde{\delta}_{2} \in \tilde{i}_{2}, \tilde{\eta}_{2} \in \tilde{f}_{2}, \\
& \left\{\left\{\left[\gamma_{1}^{L}+\gamma_{2}^{L}-\gamma_{1}^{L} \gamma_{2}^{L}, \gamma_{1}^{U}+\gamma_{2}^{U}-\gamma_{1}^{U} \gamma_{2}^{U}\right]\right\},\right. \\
& \left.\left\{\left[\delta_{1}^{L} \delta_{2}^{L}, \delta_{1}^{U} \delta_{2}^{U}\right]\right\},\left\{\left[\eta_{1}^{L} \eta_{2}^{L}, \eta_{1}^{U} \eta_{2}^{U}\right]\right\}\right\} ;
\end{aligned}
$$

(2) $\tilde{n}_{1} \otimes \tilde{n}_{2}=\left\{\tilde{t}_{1} \otimes \tilde{t}_{2}, \tilde{i}_{1} \oplus \tilde{i}_{2}, \tilde{f}_{1} \oplus \tilde{f}_{2}\right\}$

$$
\begin{aligned}
= & \tilde{\gamma}_{1 \in \tilde{f}_{1}, \tilde{\delta}_{1} \in \tilde{i}_{1}, \tilde{\eta}_{1} \in \tilde{f}_{1}, \tilde{\gamma}_{2} \in \tilde{t}_{2}, \tilde{\delta}_{2} \in \tilde{i}_{2}, \tilde{\eta}_{2} \in \tilde{f}_{2},} \\
& \left\{\left\{\left[\gamma_{1}^{L} \gamma_{2}^{L}, \gamma_{1}^{U} \gamma_{2}^{U}\right]\right\},\left\{\left[\delta_{1}^{L}+\delta_{2}^{L}-\delta_{1}^{L} \delta_{2}^{L}, \delta_{1}^{U}+\delta_{2}^{U}-\delta_{1}^{U} \delta_{2}^{U}\right]\right\},\right. \\
& \left.\left\{\left[\eta_{1}^{L}+\eta_{2}^{L}-\eta_{1}^{L} \eta_{2}^{L}, \eta_{1}^{U}+\eta_{2}^{U}-\eta_{1}^{U} \eta_{2}^{U}\right]\right\}\right\} ;
\end{aligned}
$$

(3) $\lambda \tilde{n}_{1}=\bigcup_{\tilde{\gamma}_{1} \in \tilde{t}_{1}, \tilde{\delta}_{1} \in \tilde{i}_{1}, \tilde{n}_{1} \in \tilde{f}_{1}}\left\{\left\{\left[1-\left(1-\gamma_{1}^{L}\right)^{\lambda}, 1-\left(1-\gamma_{1}^{U}\right)^{\lambda}\right]\right\},\left\{\left[\left(\delta_{1}^{L}\right)^{\lambda},\left(\delta_{1}^{U}\right)^{\lambda}\right]\right\}\right.$

$$
\left.\left\{\left[\left(\eta_{1}^{L}\right)^{\lambda},\left(\eta_{1}^{U}\right)^{\lambda}\right]\right\}\right\}
$$

(4) $\tilde{n}_{1}^{\lambda}=\bigcup_{\tilde{\gamma}_{1} \in \tilde{t}_{1}, \tilde{\delta}_{1} \in \tilde{i}_{1}, \tilde{\eta}_{1} \in \tilde{f}_{1}}\left\{\left\{\left[\left(\gamma_{1}^{L}\right)^{\lambda},\left(\gamma_{1}^{U}\right)^{\lambda}\right]\right\},\left\{\left[1-\left(1-\delta_{1}^{L}\right)^{\lambda}, 1-\left(1-\delta_{1}^{U}\right)^{\lambda}\right]\right\}\right.$

$$
\left.\left\{\left[1-\left(1-\eta_{1}^{L}\right)^{\lambda}, 1-\left(1-\eta_{1}^{U}\right)^{\lambda}\right]\right\}\right\} \text {. }
$$

\section{Correlation Coefficients of INHFSs}

The elements in an INHFE are usually given in a disorder. Therefore, for an INHFE $\tilde{n}=\{\tilde{t}, \tilde{i}, \tilde{f}\}$ it is necessary to arrange them in $\tilde{n}$ by an increasing order using Eq. (1) for the comparison between two interval numbers. Let $\tilde{\gamma}_{\sigma(k)} \in \tilde{t}(k=1,2, \ldots, l), \tilde{\delta}_{\sigma(k)} \in \tilde{i}$ $(k=1,2, \ldots, p), \tilde{\eta}_{\sigma(k)} \in \tilde{f}(k=1,2, \ldots, q)$ stand for the $k$-th smallest interval in $\tilde{n}$, and there are $\tilde{\gamma}_{\sigma(k)} \leqslant \tilde{\gamma}_{\sigma(k+1)}(k=1,2, \ldots, l-1), \tilde{\delta}_{\sigma(k)} \leqslant \tilde{\delta}_{\sigma(k+1)}(k=1,2, \ldots, p-1)$, and $\tilde{\eta}_{\sigma(k)} \leqslant \tilde{\eta}_{\sigma(k+1)}(k=1,2, \ldots, q-1)$, where $l, p, q$ are the number of interval values in $\tilde{t}, \tilde{i}, \tilde{f}$, respectively.

Let two INHFSs be $N_{1}=\left\{\left\langle x_{i}, \tilde{t}_{1}\left(x_{i}\right), \tilde{i}_{1}\left(x_{i}\right), \tilde{f}_{1}\left(x_{i}\right)\right\rangle \mid x_{i} \in X, i=1,2, \ldots, n\right\}$ and $N_{2}=\left\{\left\langle x_{i}, \tilde{t}_{2}\left(x_{i}\right), \tilde{i}_{2}\left(x_{i}\right), \tilde{f}_{2}\left(x_{i}\right)\right\rangle \mid x_{i} \in X, i=1,2, \ldots, n\right\}$. For two INHFEs $\tilde{n}_{1}\left(x_{i}\right)=$ 
$\left\{\tilde{t}_{1}\left(x_{i}\right), \tilde{i}_{1}\left(x_{i}\right), \tilde{f}_{1}\left(x_{i}\right)\right\}$ and $\tilde{n}_{2}\left(x_{i}\right)=\left\{\tilde{t}_{2}\left(x_{i}\right), \tilde{i}_{2}\left(x_{i}\right), \tilde{f}_{2}\left(x_{i}\right)\right\} \quad(i=1,2, \ldots, n)$ in the two INHFSs $N_{1}$ and $N_{2}$, the number of interval elements (numbers) in different INHFEs may be different. To compute the correlation coefficients between two INHFSs, Let $l_{i}=\max \left[l\left(\tilde{t}_{1}\left(x_{i}\right)\right), l\left(\tilde{t}_{2}\left(x_{i}\right)\right)\right], p_{i}=\max \left[l\left(\tilde{i}_{1}\left(x_{i}\right)\right), l\left(\tilde{i}_{2}\left(x_{i}\right)\right)\right]$, and $q_{i}=$ $\max \left[l\left(\tilde{f}_{1}\left(x_{i}\right)\right), l\left(\tilde{f}_{2}\left(x_{2}\right)\right)\right]$ for each $x_{i}$ in $X$, where $\left.l\left(\tilde{t}_{1}\left(x_{i}\right)\right), l\left(\tilde{t}_{2}\left(x_{i}\right)\right), l\left(\tilde{i}_{1}(x)_{1}\right), l\left(\tilde{(}_{i}\right)_{2}\left(x_{i}\right)\right)$, $l\left(\tilde{f}_{1}\left(x_{i}\right)\right)$, and $l\left(\tilde{f}_{2}\left(x_{i}\right)\right)$ represent the number of intervals in $\tilde{t}_{1}\left(x_{i}\right), \tilde{t}_{2}\left(x_{i}\right), \tilde{i}_{1}\left(x_{i}\right), \tilde{i}_{2}\left(x_{i}\right)$, $\tilde{f}_{1}\left(x_{i}\right)$, and $\tilde{f}_{2}\left(x_{i}\right)$, respectively. When $l\left(\tilde{t}_{1}\left(x_{i}\right)\right) \neq l\left(\tilde{t}_{2}\left(x_{i}\right)\right)$ or $l\left(\tilde{i}_{1}\left(x_{i}\right)\right) \neq l\left(\tilde{i}_{2}\left(x_{i}\right)\right)$ or $l\left(\tilde{f}_{1}\left(x_{i}\right)\right) \neq l\left(\tilde{f}_{2}\left(x_{i}\right)\right)$, one can make them having the same number of elements through adding some elements to less number of elements in the INHFE. For example, if there are fewer elements in $\tilde{t}_{1}\left(x_{i}\right)$ than in $\tilde{t}_{2}\left(x_{i}\right)$, an extension of $\tilde{t}_{1}\left(x_{i}\right)$ should be considered optimistically by repeating its maximum element until has the same length as $\tilde{t}_{2}\left(x_{i}\right)$. Thus, we use similar method to realize the same length of between $\tilde{i}_{1}\left(x_{i}\right)$ and $\tilde{i}_{2}\left(x_{i}\right)$ or between $\tilde{f}_{1}\left(x_{i}\right)$ and $\tilde{f}_{2}\left(x_{i}\right)$.

Similar to the existing works (Chen et al., 2013a), we can define the informational energy for INHFSs and the corresponding correlation.

Definition 12. For an INHFS $N_{1}=\left\{\left\langle x_{i}, \tilde{t}_{1}\left(x_{i}\right), \tilde{i}_{1}\left(x_{i}\right), \tilde{f}_{1}\left(x_{i}\right)\right\rangle \mid x_{i} \in X, i=1,2, \ldots, n\right\}$, the informational energy for the INHFS $N_{1}$ is defined as

$$
\begin{aligned}
E_{I N H F S}\left(N_{1}\right)= & \sum_{i=1}^{n}\left\{\frac{1}{l_{i}} \sum_{k=1}^{l_{i}}\left[\left(\gamma_{1 i \sigma(k)}^{L}\right)^{2}+\left(\gamma_{1 i \sigma(k)}^{U}\right)^{2}\right]\right. \\
& +\frac{1}{p_{i}} \sum_{k=1}^{p_{i}}\left[\left(\delta_{1 i \sigma(k)}^{L}\right)^{2}+\left(\delta_{1 i \sigma(k)}^{U}\right)^{2}\right] \\
& \left.+\frac{1}{q_{i}} \sum_{k=1}^{q_{i}}\left[\left(\eta_{1 i \sigma(k)}^{L}\right)^{2}+\left(\eta_{1 i \sigma(k)}^{U}\right)^{2}\right]\right\}
\end{aligned}
$$

where $\tilde{\gamma}_{1 i \sigma(k)}=\left[\gamma_{1 i \sigma(k)}^{L}, \gamma_{1 i \sigma(k)}^{U}\right] \in \tilde{t}_{1}\left(x_{i}\right)\left(k=1,2, \ldots, l_{i} ; i=1,2, \ldots, n\right), \tilde{\delta}_{1 i \sigma(k)}=$ $\left[\delta_{1 i \sigma(k)}^{L}, \delta_{1 i \sigma(k)}^{U}\right] \in \tilde{i}_{1}\left(x_{i}\right) \quad\left(k=1,2, \ldots, p_{i} ; i=1,2, \ldots, n\right)$, and $\tilde{\eta}_{1 i \sigma(k)}=\left[\eta_{1 i \sigma(k)}^{L}\right.$, $\left.\eta_{1 i \sigma(k)}^{U}\right] \in \tilde{f}_{1}\left(x_{i}\right)\left(k=1,2, \ldots, q_{i} ; i=1,2, \ldots, n\right)$ and $l_{i}, p_{i}, q_{i}$ are the number of intervals in $\tilde{t}_{1}\left(x_{i}\right), \tilde{i}_{1}\left(x_{i}\right), \tilde{f}_{1}\left(x_{i}\right)$, respectively.

Definition 13. For two INHFSs $N_{1}$ and $N_{2}$, their correlation is defined as

$$
\begin{aligned}
C_{I N H F S 1}\left(N_{1}, N_{2}\right)= & \sum_{i=1}^{n}\left\{\frac{1}{l_{i}} \sum_{k=1}^{l_{i}}\left[\gamma_{1 i \sigma(k)}^{L} \cdot \gamma_{2 i \sigma(k)}^{L}+\gamma_{1 i \sigma(k)}^{U} \cdot \gamma_{2 i \sigma(k)}^{U}\right]\right. \\
& +\frac{1}{p_{i}} \sum_{k=1}^{p_{i}}\left[\delta_{1 i \sigma(k)}^{L} \cdot \delta_{2 i \sigma(k)}^{L}+\delta_{1 i \sigma(k)}^{U} \cdot \delta_{2 i \sigma(k)}^{U}\right] \\
& \left.+\frac{1}{q_{i}} \sum_{k=1}^{q_{i}}\left[\eta_{1 i \sigma(k)}^{L} \cdot \eta_{2 i \sigma(k)}^{L}+\eta_{1 i \sigma(k)}^{U} \cdot \eta_{2 i \sigma(k)}^{U}\right]\right\}
\end{aligned}
$$


where $\tilde{\gamma}_{1 i \sigma(k)}=\left[\gamma_{1 i \sigma(k)}^{L}, \gamma_{1 i \sigma(k)}^{U}\right] \in \tilde{t}_{1}\left(x_{i}\right)$, and $\tilde{\gamma}_{2 i \sigma(k)}=\left[\gamma_{2 i \sigma(k)}^{L}, \gamma_{2 i \sigma(k)}^{U}\right] \in \tilde{t}_{2}\left(x_{i}\right)$, $\left(k=1,2, \ldots, l_{i} ; i=1,2, \ldots, n\right), \tilde{\delta}_{1 i \sigma(k)}=\left[\delta_{1 i \sigma(k)}^{L}, \delta_{1 i \sigma(k)}^{U}\right] \in \tilde{i}_{1}\left(x_{i}\right)$, and $\tilde{\delta}_{2 i \sigma(k)}=$ $\left[\delta_{2 i \sigma(k)}^{L}, \delta_{2 i \sigma(k)}^{U}\right] \in \tilde{i}_{2}\left(x_{i}\right),\left(k=1,2, \ldots, p_{i} ; i=1,2, \ldots, n\right), \tilde{\eta}_{1 i \sigma(k)}=\left[\eta_{1 i \sigma(k)}^{L}, \eta_{1 i \sigma(k)}^{U}\right] \in$ $\tilde{f}_{1}\left(x_{i}\right)$, and $\tilde{\eta}_{2 i \sigma(k)}=\left[\eta_{2 i \sigma(k)}^{L}, \eta_{2 i \sigma(k)}^{U}\right] \in \tilde{f}_{2}\left(x_{i}\right),\left(k=1,2, \ldots, q_{i} ; i=1,2, \ldots, n\right)$, and $l_{i}, p_{i}, q_{i}$ are the number of intervals in $\tilde{t}_{1}\left(x_{i}\right)$ and $\tilde{t}_{2}\left(x_{i}\right), \tilde{i}_{1}\left(x_{i}\right)$ and $\tilde{i}_{2}\left(x_{i}\right), \tilde{f}_{1}\left(x_{i}\right)$ and $\tilde{f}_{2}\left(x_{i}\right)$, respectively.

It is obvious that the correlation (5) satisfies the following properties:

(1) $C_{I N H F S I}\left(N_{1}, N_{1}\right)=E_{I N H F S}\left(N_{1}\right)$;

(2) $C_{I N H F S I}\left(N_{1}, N_{2}\right)=C_{I N H F S I}\left(N_{2}, N_{1}\right)$.

According to Definitions 12 and 13, we derive a correlation coefficient between the INHFSs $N_{1}$ and $N_{2}$.

$$
\begin{aligned}
R_{I N H F S I}\left(N_{1}, N_{2}\right)= & \frac{C_{I N H F S I}\left(N_{1}, N_{2}\right)}{\left[C_{I N H F S I}\left(N_{1}, N_{1}\right)\right]^{1 / 2} \cdot\left[C_{I N H F S I}\left(N_{2}, N_{2}\right)\right]^{1 / 2}} \\
= & \frac{\left(\begin{array}{c}
\sum_{i=1}^{n}\left\{\frac{1}{l_{i}} \sum_{k=1}^{l_{i}}\left[\gamma_{1 i \sigma(k)}^{L} \cdot \gamma_{2 i \sigma(k)}^{L}+\gamma_{1 i \sigma(k)}^{U} \cdot \gamma_{2 i \sigma(k)}^{U}\right]\right. \\
+ \\
p_{i} \sum_{k=1}^{p_{i}}\left[\delta_{1 i \sigma(k)}^{L} \cdot \delta_{2 i \sigma(k)}^{L}+\delta_{1 i \sigma(k)}^{U} \cdot \delta_{2 i \sigma(k)}^{U}\right] \\
\left.+\frac{1}{q_{i}} \sum_{k=1}^{q_{i}}\left[\eta_{1 i \sigma(k)}^{L} \cdot \eta_{2 i \sigma(k)}^{L}+\eta_{1 i \sigma(k)}^{U} \cdot \eta_{2 i \sigma(k)}^{U}\right]\right\}
\end{array}\right)}{\left(\begin{array}{c}
\left\{\sum _ { i = 1 } ^ { n } \left\{\frac{1}{l_{i}} \sum_{k=1}^{l_{i}}\left[\left(\gamma_{1 i \sigma(k)}^{L}\right)^{2}+\left(\gamma_{1 i \sigma(k)}^{U}\right)^{2}\right]\right.\right. \\
+\frac{1}{p_{i}} \sum_{k=1}^{p_{i}}\left[\left(\delta_{1 i \sigma(k)}^{L}\right)^{2}+\left(\delta_{1 i \sigma(k)}^{U}\right)^{2}\right] \\
\left.\left.+\frac{1}{q_{i}} \sum_{k=1}^{q_{i}}\left[\left(\eta_{1 i \sigma(k)}^{L}\right)^{2}+\left(\eta_{1 i \sigma(k)}^{U}\right)^{2}\right]\right\}\right\}^{1 / 2} \\
\times\left\{\sum _ { i = 1 } ^ { n } \left\{\frac{1}{l_{i}} \sum_{k=1}^{l_{i}}\left[\left(\gamma_{2 i \sigma(k)}^{L}\right)^{2}+\left(\gamma_{2 i \sigma(k)}^{U}\right)^{2}\right]\right.\right. \\
+\frac{1}{p_{i}} \sum_{k=1}^{p_{i}}\left[\left(\delta_{2 i \sigma(k)}^{L}\right)^{2}+\left(\delta_{2 i \sigma(k)}^{U}\right)^{2}\right] \\
\left.\left.+\frac{1}{q_{i}} \sum_{k=1}^{q_{i}}\left[\left(\eta_{2 i \sigma(k)}^{L}\right)^{2}+\left(\eta_{2 i \sigma(k)}^{U}\right)^{2}\right]\right\}\right\}^{1 / 2}
\end{array}\right)} .
\end{aligned}
$$

Theorem 1. The correlation coefficient $R_{I N H F S I}\left(N_{1}, N_{2}\right)$ satisfies the following properties:

(1) $R_{I N H F S I}\left(N_{1}, N_{2}\right)=R_{I N H F S I}\left(N_{2}, N_{1}\right)$;

(2) $0 \leqslant R_{I N H F S I}\left(N_{1}, N_{2}\right) \leqslant 1$;

(3) $R_{I N H F S I}\left(N_{1}, N_{2}\right)=1$, if $N_{1}=N_{2}$.

Proof. (1) It is straightforward.

(2) The inequality $R_{I N H F S I}\left(N_{1}, N_{2}\right) \geqslant 0$ is obvious. Below let us prove $R_{I N H F S I}\left(N_{1}, N_{2}\right) \leqslant 1$ :

According to the Cauchy-Schwarz inequality:

$$
\left(x_{1} y_{1}+x_{2} y_{2}+\cdots+x_{n} y_{n}\right)^{2} \leqslant\left(x_{1}^{2}+x_{2}^{2}+\cdots+x_{n}^{2}\right) \cdot\left(y_{1}^{2}+y_{2}^{2}+\ldots+y_{n}^{2}\right),
$$


where $\left(x_{1}, x_{2}, \ldots, x_{n}\right) \in R^{n}$ and $\left(y_{1}, y_{2}, \ldots, y_{n}\right) \in R^{n}$. Then, there is the following inequality:

$$
\left(\sum_{i=1}^{n}\left(x_{i} y_{i}\right)\right)^{2} \leqslant \sum_{i=1}^{n}\left(x_{i}^{2}\right) \sum_{i=1}^{n}\left(y_{i}^{2}\right) .
$$

Then, according to Eq. (5) and the above inequality, we have

$$
\begin{aligned}
& {\left[C_{I N H F S 1}\left(N_{1}, N_{2}\right)\right]^{2}} \\
& =\left(\begin{array}{c}
\sum_{i=1}^{n}\left\{\frac{1}{l_{i}} \sum_{k=1}^{l_{i}}\left[\gamma_{1 i \sigma(k)}^{L} \cdot \gamma_{2 i \sigma(k)}^{L}+\gamma_{1 i \sigma(k)}^{U} \cdot \gamma_{2 i \sigma(k)}^{U}\right]\right. \\
+\frac{1}{p_{i}} \sum_{k=1}^{p_{i}}\left[\delta_{1 i \sigma(k)}^{L} \cdot \delta_{2 i \sigma(k)}^{L}+\delta_{1 i \sigma(k)}^{U} \cdot \delta_{2 i \sigma(k)}^{U}\right] \\
\left.+\frac{1}{q_{i}} \sum_{k=1}^{q_{i}}\left[\eta_{1 i \sigma(k)}^{L} \cdot \eta_{2 i \sigma(k)}^{L}+\eta_{1 i \sigma(k)}^{U} \cdot \eta_{2 i \sigma(k)}^{U}\right]\right\}
\end{array}\right)^{2} \\
& \left\{\begin{array}{c}
\left\{\left[\sum_{k=1}^{l_{1}} \frac{\gamma_{11 \sigma(k)}^{L} \cdot \gamma_{21 \sigma(k)}^{L}}{\sqrt{l_{1}} \cdot \sqrt{l_{1}}}+\sum_{k=1}^{l_{1}} \frac{\gamma_{11 \sigma(k)}^{U} \cdot \gamma_{21 \sigma(k)}^{U}}{\sqrt{l_{1}} \cdot \sqrt{l_{1}}}\right]\right. \\
+\left[\sum_{k=1}^{p_{1}} \frac{\delta_{11 \sigma(k)}^{L} \cdot \delta_{21 \sigma(k)}^{L}}{\sqrt{p_{1}} \cdot \sqrt{p_{1}}}+\sum_{k=1}^{p_{1}} \frac{\delta_{11 \sigma(k)}^{U} \cdot \delta_{21 \sigma(k)}^{U}}{\sqrt{p_{1}} \cdot \sqrt{p_{1}}}\right] \\
+\left[\sum_{k}^{q_{1}} \frac{\eta_{11 \sigma(k)}^{L} \cdot \eta_{21 \sigma(k)}^{L}}{\sqrt{q_{1}} \cdot \sqrt{q_{1}}}+\sum_{k 1}^{q_{1}} \frac{\eta_{11 \sigma(k)}^{U} \cdot \eta_{21 \sigma(k)}^{U}}{\sqrt{q_{1}} \cdot \sqrt{q}}\right]
\end{array}\right. \\
& +\left[\sum_{k=1}^{q_{1}} \frac{\eta_{11 \sigma(k)}^{L} \cdot \eta_{21 \sigma(k)}^{L}}{\sqrt{q_{1}} \cdot \sqrt{q_{1}}}+\sum_{k=1}^{q_{1}} \frac{\eta_{11 \sigma(k)}^{U} \cdot \eta_{21 \sigma(k)}^{U}}{\sqrt{q_{1}} \cdot \sqrt{q_{1}}}\right] \\
& +\left[\sum_{k=1}^{l_{2}} \frac{\gamma_{12 \sigma(k)}^{L} \cdot \gamma_{22 \sigma(k)}^{L}}{\sqrt{l_{2}} \cdot \sqrt{l_{2}}}+\sum_{k=1}^{l_{2}} \frac{\gamma_{12 \sigma(k)}^{U} \cdot \gamma_{22 \sigma(k)}^{U}}{\sqrt{l_{2}} \cdot \sqrt{l_{2}}}\right] \\
& =\quad+\left[\sum_{k=1}^{p_{2}} \frac{\delta_{12 \sigma(k)}^{L} \cdot \delta_{22 \sigma(k)}^{L}}{\sqrt{p_{2}} \cdot \sqrt{p_{2}}}+\sum_{k=1}^{p_{2}} \frac{\delta_{12 \sigma(k)}^{U} \cdot \delta_{22 \sigma(k)}^{U}}{\sqrt{p_{2}} \cdot \sqrt{p_{2}}}\right] \\
& +\left[\sum_{k=1}^{q_{2}} \frac{\eta_{12 \sigma(k)}^{L} \cdot \eta_{22 \sigma(k)}^{L}}{\sqrt{q_{2}} \cdot \sqrt{q_{2}}}+\sum_{k=1}^{q_{2}} \frac{\eta_{12 \sigma(k)}^{U} \cdot \eta_{22 \sigma(k)}^{U}}{\sqrt{q_{2}} \cdot \sqrt{q_{2}}}\right] \\
& +\cdots+\left[\sum_{k=1}^{l_{n}} \frac{\gamma_{1 n \sigma(k)}^{L} \cdot \gamma_{2 n \sigma(k)}^{L}}{\sqrt{l_{n}} \cdot \sqrt{l_{n}}}+\sum_{k=1}^{l_{n}} \frac{\gamma_{1 n \sigma(k)}^{U} \cdot \gamma_{2 n \sigma(k)}^{U}}{\sqrt{l_{n}} \cdot \sqrt{l_{n}}}\right] \\
& +\left[\sum_{k=1}^{p_{n}} \frac{\delta_{1 n \sigma(k)}^{L} \cdot \delta_{2 n \sigma(k)}^{L}}{\sqrt{p_{n}} \cdot \sqrt{p_{n}}}+\sum_{k=1}^{p_{n}} \frac{\delta_{1 n \sigma(k)}^{U} \cdot \delta_{2 n \sigma(k)}^{U}}{\sqrt{p_{n}} \cdot \sqrt{p_{n}}}\right] \\
& \left.+\left[\sum_{k=1}^{q_{n}} \frac{\eta_{1 n \sigma(k)}^{L} \cdot \eta_{2 n \sigma(k)}^{L}}{\sqrt{q_{n}} \cdot \sqrt{q_{n}}}+\sum_{k=1}^{q_{n}} \frac{\eta_{1 n \sigma(k)}^{U} \cdot \eta_{2 n \sigma(k)}^{U}}{\sqrt{q_{n}} \cdot \sqrt{q_{n}}}\right]\right\} \\
& =\left(\begin{array}{c}
\left\{\sum_{k=1}^{l_{1}} \frac{\gamma_{11 \sigma(k)}^{L} \cdot \gamma_{21 \sigma(k)}^{L}}{\sqrt{l_{1}} \cdot \sqrt{l_{1}}}+\sum_{k=1}^{p_{1}} \frac{\delta_{11 \sigma(k)}^{L} \cdot \delta_{21 \sigma(k)}^{L}}{\sqrt{p_{1}} \cdot \sqrt{p_{1}}}+\sum_{k=1}^{q_{1}} \frac{\eta_{11 \sigma(k)}^{L} \cdot \eta_{21 \sigma(k)}^{L}}{\sqrt{q_{1}} \cdot \sqrt{q_{1}}}\right. \\
\quad+\sum_{k=1}^{l_{1}} \frac{\gamma_{11 \sigma(k)}^{U} \cdot \gamma_{21 \sigma(k)}^{U}}{\sqrt{l_{1}} \cdot \sqrt{l_{1}}}+\sum_{k=1}^{p_{1}} \frac{\delta_{11 \sigma(k)}^{U} \cdot \delta_{21 \sigma(k)}^{U}}{\sqrt{p_{1}} \cdot \sqrt{p_{1}}}+\sum_{k=1}^{q_{1}} \frac{\eta_{11 \sigma(k)}^{U} \cdot \eta_{21 \sigma(k)}^{U}}{\sqrt{q_{1}} \cdot \sqrt{q_{1}}} \\
\quad+\sum_{k=1}^{l_{2}} \frac{\gamma_{12 \sigma(k)}^{L} \cdot \gamma_{22 \sigma(k)}^{L}}{\sqrt{l_{2}} \cdot \sqrt{l_{2}}}+\sum_{k=1}^{p_{2}} \frac{\delta_{12 \sigma(k)}^{L} \cdot \delta_{22 \sigma(k)}^{L}}{\sqrt{p_{2}} \cdot \sqrt{p_{2}}}+\sum_{k=1}^{q_{2}} \frac{\eta_{12 \sigma(k)}^{L} \cdot \eta_{22 \sigma(k)}^{L}}{\sqrt{q_{2}} \cdot \sqrt{q_{2}}} \\
\quad+\sum_{k=1}^{l_{2}} \frac{\gamma_{12 \sigma(k)}^{U} \cdot \gamma_{22 \sigma(k)}^{U}}{\sqrt{l_{2}} \cdot \sqrt{l_{2}}}+\sum_{k=1}^{p_{2}} \frac{\delta_{12 \sigma(k)}^{U} \cdot \delta_{22 \sigma(k)}^{U}}{\sqrt{p_{2}} \cdot \sqrt{p_{2}}}+\sum_{k=1}^{q_{2}} \frac{\eta_{12 \sigma(k)}^{U} \cdot \eta_{22 \sigma(k)}^{U}}{\sqrt{q_{2}} \cdot \sqrt{q_{2}}} \\
\quad+\cdots+\sum_{k=1}^{l_{n}} \frac{\gamma_{1 n \sigma(k)}^{L} \cdot \gamma_{2 n \sigma(k)}^{L}}{\sqrt{l_{n}} \cdot \sqrt{l_{n}}}+\sum_{k=1}^{p_{n}} \frac{\delta_{1 n \sigma(k)}^{L} \cdot \delta_{2 n \sigma(k)}^{L}}{\sqrt{p_{n}} \cdot \sqrt{p_{n}}}+\sum_{k=1}^{q_{n}} \frac{\eta_{1 n \sigma(k)}^{L} \cdot \eta_{2 n \sigma(k)}^{L}}{\sqrt{q_{n}} \cdot \sqrt{q_{n}}} \\
\left.\quad+\sum_{k=1}^{l_{n}} \frac{\gamma_{1 n \sigma(k)}^{U} \cdot \gamma_{2 n \sigma(k)}^{U}}{\sqrt{l_{n}} \cdot \sqrt{l_{n}}}+\sum_{k=1}^{p_{n}} \frac{\delta_{1 n \sigma(k)}^{U} \cdot \delta_{2 n \sigma(k)}^{U}}{\sqrt{p_{n}} \cdot \sqrt{p_{n}}}+\sum_{k=1}^{q_{n}} \frac{\eta_{1 n \sigma(k)}^{U} \cdot \eta_{2 n \sigma(k)}^{U}}{\sqrt{q_{n}} \cdot \sqrt{q_{n}}}\right\}
\end{array}\right)^{2}
\end{aligned}
$$


Therefore, we can obtain

$$
\begin{aligned}
& {\left[C_{I N H F S I}\left(N_{1}, N_{2}\right)\right]^{2}} \\
& \leqslant\left\{\sum_{k=1}^{l_{1}} \frac{1}{l_{1}}\left(\gamma_{11 \sigma(k)}^{L}\right)^{2}+\sum_{k=1}^{p_{1}} \frac{1}{p_{1}}\left(\delta_{11 \sigma(k)}^{L}\right)^{2}+\sum_{k=1}^{q_{1}} \frac{1}{q_{1}}\left(\eta_{11 \sigma(k)}^{L}\right)^{2}\right. \\
& +\sum_{k=1}^{l_{2}} \frac{1}{l_{2}}\left(\gamma_{12 \sigma(k)}^{L}\right)^{2}+\sum_{k=1}^{p_{2}} \frac{1}{p_{2}}\left(\delta_{12 \sigma(k)}^{L}\right)^{2}+\sum_{k=1}^{q_{2}} \frac{1}{q_{2}}\left(\eta_{12 \sigma(k)}^{L}\right)^{2}+\ldots \\
& +\sum_{k=1}^{l_{n}} \frac{1}{l_{n}}\left(\gamma_{1 n \sigma(k)}^{L}\right)^{2}+\sum_{k=1}^{p_{n}} \frac{1}{p_{n}}\left(\delta_{1 n \sigma(k)}^{L}\right)^{2}+\sum_{k=1}^{q_{n}} \frac{1}{q_{n}}\left(\eta_{1 n \sigma(k)}^{L}\right)^{2} \\
& +\sum_{k=1}^{l_{1}} \frac{1}{l_{1}}\left(\gamma_{11 \sigma(k)}^{U}\right)^{2}+\sum_{k=1}^{p_{1}} \frac{1}{p_{1}}\left(\delta_{11 \sigma(k)}^{U}\right)^{2}+\sum_{k=1}^{q_{1}} \frac{1}{q_{1}}\left(\eta_{11 \sigma(k)}^{U}\right)^{2} \\
& \left.+\sum_{k=1}^{l_{2}} \frac{1}{l_{2}}\left(\gamma_{12 \sigma(k)}^{U}\right)^{(} 2\right)+\sum_{k=1}^{p_{2}} \frac{1}{p_{2}}\left(\delta_{12 \sigma(k)}^{U}\right)^{2}+\sum_{k=1}^{q_{2}} \frac{1}{q_{2}}\left(\eta_{12 \sigma(k)}^{U}\right)^{2}+\cdots \\
& \left.+\sum_{k=1}^{l_{n}} \frac{1}{l_{n}}\left(\gamma_{1 n \sigma(k)}^{U}\right)^{2}+\sum_{k=1}^{p_{n}} \frac{1}{p_{n}}\left(\delta_{1 n \sigma(k)}^{U}\right)^{2}+\sum_{k=1}^{q_{n}} \frac{1}{q_{n}}\left(\eta_{1 n \sigma(k)}^{U}\right)^{2}\right\} \\
& \times\left\{\sum_{k=1}^{l_{1}} \frac{1}{l_{1}}\left(\gamma_{21 \sigma(k)}^{L}\right)^{2}+\sum_{k=1}^{p_{1}} \frac{1}{p_{1}}\left(\delta_{21 \sigma(k)}^{L}\right)^{2}+\sum_{k=1}^{q_{1}} \frac{1}{q_{1}}\left(\eta_{21 \sigma(k)}^{L}\right)^{2}\right. \\
& +\sum_{k=1}^{l_{2}} \frac{1}{l_{2}}\left(\gamma_{22 \sigma(k)}^{L}\right)^{2}+\sum_{k=1}^{p_{2}} \frac{1}{p_{2}}\left(\delta_{22 \sigma(k)}^{L}\right)^{2}+\sum_{k=1}^{q_{2}} \frac{1}{q_{2}}\left(\eta_{22 \sigma(k)}^{L}\right)^{2}+\cdots \\
& +\sum_{k=1}^{l_{n}} \frac{1}{l_{n}}\left(\gamma_{2 n \sigma(k)}^{L}\right)^{2}+\sum_{k=1}^{p_{n}} \frac{1}{p_{n}}\left(\delta_{2 n \sigma(k)}^{L}\right)^{2}+\sum_{k=1}^{q_{n}} \frac{1}{q_{n}}\left(\eta_{2 n \sigma(k)}^{L}\right)^{2} \\
& +\sum_{k=1}^{l_{1}} \frac{1}{l_{1}}\left(\gamma_{21 \sigma(k)}^{U}\right)^{2}+\sum_{k=1}^{p_{1}} \frac{1}{p_{1}}\left(\delta_{21 \sigma(k)}^{U}\right)^{2}+\sum_{k=1}^{q_{1}} \frac{1}{q_{1}}\left(\eta_{21 \sigma(k)}^{U}\right)^{2} \\
& +\sum_{k=1}^{l_{2}} \frac{1}{l_{2}}\left(\gamma_{22 \sigma(k)}^{U}\right)^{2}+\sum_{k=1}^{p_{2}} \frac{1}{p_{2}}\left(\delta_{22 \sigma(k)}^{U}\right)^{2}+\sum_{k=1}^{q_{2}} \frac{1}{q_{2}}\left(\eta_{22 \sigma(k)}^{U}\right)^{2}+\cdots \\
& \left.+\sum_{k=1}^{l_{n}} \frac{1}{l_{n}}\left(\gamma_{2 n \sigma(k)}^{U}\right)^{2}+\sum_{k=1}^{p_{n}} \frac{1}{p_{n}}\left(\delta_{2 n \sigma(k)}^{U}\right)^{2}+\sum_{k=1}^{q_{n}} \frac{1}{q_{n}}\left(\eta_{2 n \sigma(k)}^{U}\right)^{2}\right\} \\
& =\left\{\left[\sum_{k=1}^{l_{1}} \frac{1}{l_{1}}\left(\gamma_{11 \sigma(k)}^{L}\right)^{2}+\sum_{k=1}^{l_{1}} \frac{1}{l_{1}}\left(\gamma_{11 \sigma(k)}^{U}\right)^{2}\right]\right.
\end{aligned}
$$




$$
\begin{aligned}
& +\left[\sum_{k=1}^{p_{1}} \frac{1}{p_{1}}\left(\delta_{11 \sigma(k)}^{L}\right)^{2}+\sum_{k=1}^{p_{1}} \frac{1}{p_{1}}\left(\delta_{11 \sigma(k)}^{U}\right)^{2}\right] \\
& +\left[\sum_{k=1}^{q_{1}} \frac{1}{q_{1}}\left(\eta_{11 \sigma(k)}^{L}\right)^{2}+\sum_{k=1}^{q_{1}} \frac{1}{q_{1}}\left(\eta_{11 \sigma(k)}^{U}\right)^{2}\right] \\
& +\left[\sum_{k=1}^{l_{2}} \frac{1}{l_{2}}\left(\gamma_{12 \sigma(k)}^{L}\right)^{2}+\sum_{k=1}^{l_{2}} \frac{1}{l_{2}}\left(\gamma_{12 \sigma(k)}^{U}\right)^{2}\right] \\
& +\left[\sum_{k=1}^{p_{2}} \frac{1}{p_{2}}\left(\delta_{12 \sigma(k)}^{L}\right)^{2}+\sum_{k=1}^{p_{2}} \frac{1}{p_{2}}\left(\delta_{12 \sigma(k)}^{U}\right)^{2}\right] \\
& +\left[\sum_{k=1}^{q_{2}} \frac{1}{q_{2}}\left(\eta_{12 \sigma(k)}^{L}\right)^{2}+\sum_{k=1}^{q_{2}} \frac{1}{q_{2}}\left(\eta_{12 \sigma(k)}^{U}\right)^{2}+\cdots\right] \\
& +\left[\sum_{k=1}^{l_{n}} \frac{1}{l_{n}}\left(\gamma_{1 n \sigma(k)}^{L}\right)^{2}+\sum_{k=1}^{l_{n}} \frac{1}{l_{n}}\left(\gamma_{1 n \sigma(k)}^{U}\right)^{2}\right] \\
& +\left[\sum_{k=1}^{p_{n}} \frac{1}{p_{n}}\left(\delta_{1 n \sigma(k)}^{L}\right)^{2}+\sum_{k=1}^{p_{n}} \frac{1}{p_{n}}\left(\delta_{1 n \sigma(k)}^{U}\right)^{2}\right] \\
& \left.+\left[\sum_{k=1}^{q_{n}} \frac{1}{q_{n}}\left(\eta_{1 n \sigma(k)}^{L}\right)^{2}+\sum_{k=1}^{q_{n}} \frac{1}{q_{n}}\left(\eta_{1 n \sigma(k)}^{U}\right)^{2}\right]\right\} \\
& \times\left\{\left[\sum_{k=1}^{l_{1}} \frac{1}{l_{1}}\left(\gamma_{21 \sigma(k)}^{L}\right)^{2}+\sum_{k=1}^{l_{1}} \frac{1}{l_{1}}\left(\gamma_{21 \sigma(k)}^{U}\right)^{2}\right]\right. \\
& +\left[\sum_{k=1}^{p_{1}} \frac{1}{p_{1}}\left(\delta_{21 \sigma(k)}^{L}\right)^{2}+\sum_{k=1}^{p_{1}} \frac{1}{p_{1}}\left(\delta_{21 \sigma(k)}^{U}\right)^{2}\right] \\
& +\left[\sum_{k=1}^{q_{1}} \frac{1}{q_{1}}\left(\eta_{21 \sigma(k)}^{L}\right)^{2}+\sum_{k=1}^{q_{1}} \frac{1}{q_{1}}\left(\eta_{21 \sigma(k)}^{U}\right)^{2}\right] \\
& +\left[\sum_{k=1}^{l_{2}} \frac{1}{l_{2}}\left(\gamma_{22 \sigma(k)}^{L}\right)^{2}+\sum_{k=1}^{l_{2}} \frac{1}{l_{2}}\left(\gamma_{22 \sigma(k)}^{U}\right)^{2}\right] \\
& +\left[\sum_{k=1}^{p_{2}} \frac{1}{p_{2}}\left(\delta_{22 \sigma(k)}^{L}\right)^{2}+\sum_{k=1}^{p_{2}} \frac{1}{p_{2}}\left(\delta_{22 \sigma(k)}^{U}\right)^{2}\right] \\
& +\left[\sum_{k=1}^{q_{2}} \frac{1}{q_{2}}\left(\eta_{22 \sigma(k)}^{L}\right)^{2}+\sum_{k=1}^{q_{2}} \frac{1}{q_{2}}\left(\eta_{22 \sigma(k)}^{U}\right)^{2}+\cdots\right]
\end{aligned}
$$




$$
\begin{aligned}
& +\left[+\sum_{k=1}^{l_{n}} \frac{1}{l_{n}}\left(\gamma_{2 n \sigma(k)}^{L}\right)^{2}+\sum_{k=1}^{l_{n}} \frac{1}{l_{n}}\left(\gamma_{2 n \sigma(k)}^{U}\right)^{2}\right] \\
& +\left[\sum_{k=1}^{p_{n}} \frac{1}{p_{n}}\left(\delta_{2 n \sigma(k)}^{L}\right)^{2}+\sum_{k=1}^{p_{n}} \frac{1}{p_{n}}\left(\delta_{2 n \sigma(k)}^{U}\right)^{2}\right] \\
& \left.+\left[\sum_{k=1}^{q_{n}} \frac{1}{q_{n}}\left(\eta_{2 n \sigma(k)}^{L}\right)^{2}+\sum_{k=1}^{q_{n}} \frac{1}{q_{n}}\left(\eta_{2 n \sigma(k)}^{U}\right)^{2}\right]\right\} \\
& =\sum_{i=1}^{n}\left\{\left[\sum_{k=1}^{l_{i}} \frac{1}{l_{i}}\left(\gamma_{1 i \sigma(k)}^{L}\right)^{2}+\sum_{k=1}^{l_{i}} \frac{1}{l_{i}}\left(\gamma_{1 i \sigma(k)}^{U}\right)^{2}\right]\right. \\
& +\left[\sum_{k=1}^{p_{i}} \frac{1}{p_{i}}\left(\delta_{1 i \sigma(k)}^{L}\right)^{2}+\sum_{k=1}^{p_{i}} \frac{1}{p_{i}}\left(\delta_{1 i \sigma(k)}^{U}\right)^{2}\right] \\
& \left.+\left[\sum_{k=1}^{q_{i}} \frac{1}{q_{i}}\left(\eta_{1 i \sigma(k)}^{L}\right)^{2}+\sum_{k=1}^{q_{i}} \frac{1}{q_{i}}\left(\eta_{1 i \sigma(k)}^{U}\right)^{2}\right]\right\} \\
& +\sum_{i=1}^{n}\left\{\left[\sum_{k=1}^{l_{i}} \frac{1}{l_{i}}\left(\gamma_{2 i \sigma(k)}^{L}\right)^{2}+\sum_{k=1}^{l_{i}} \frac{1}{l_{i}}\left(\gamma_{2 i \sigma(k)}^{U}\right)^{2}\right]\right. \\
& +\left[\sum_{k=1}^{p_{i}} \frac{1}{p_{i}}\left(\delta_{2 i \sigma(k)}^{L}\right)^{2}+\sum_{k=1}^{p_{i}} \frac{1}{p_{i}}\left(\delta_{2 i \sigma(k)}^{U}\right)^{2}\right] \\
& \left.+\left[\sum_{k=1}^{q_{i}} \frac{1}{q_{i}}\left(\eta_{2 i \sigma(k)}^{L}\right)^{2}+\sum_{k=1}^{q_{i}} \frac{1}{q_{i}}\left(\eta_{2 i \sigma(k)}^{U}\right)^{2}\right]\right\} \\
& C_{I N H F S I}\left(N_{1}, N_{1}\right) \cdot C_{I N H F S I}\left(N_{2}, N_{2}\right) .
\end{aligned}
$$

Therefore

$$
C_{\text {INHFSI }}\left(N_{1}, N_{2}\right) \leqslant\left[C_{\text {INHFSI }}\left(N_{1}, N_{1}\right)\right]^{1 / 2}\left[C_{\text {INHFSI }}\left(N_{2}, N_{2}\right)\right]^{1 / 2} .
$$

Thus, $0 \leqslant R_{I N H F S I}\left(N_{1}, N_{2}\right) \leqslant 1$.

(3) $N_{1}=N_{2} \Rightarrow \gamma_{1 i \sigma(k)}^{L}=\gamma_{2 i \sigma(k)}^{L}, \gamma_{1 i \sigma(k)}^{U}=\gamma_{2 i \sigma(k)}^{U}, \delta_{1 i \sigma(k)}^{L}=\delta_{2 i \sigma(k)}^{L}, \delta_{1 i \sigma(k)}^{U}=\delta_{2 i \sigma(k)}^{U}$, $\eta_{1 i \sigma(k)}^{L}=\eta_{2 i \sigma(k)}^{L}$, and $\eta_{1 i \sigma(k)}^{U}=\eta_{2 i \sigma(k)}^{U}$ for any $x_{i} \in X \Rightarrow R_{I N H F S I}\left(N_{1}, N_{2}\right)=1$.

As a further generalization of the correlation coefficient between IVHFSs for Eq. (3), we give another formula of the correlation coefficient of INHFSs.

Definition 14. For two INHFSs $N_{1}$ and $N_{2}$ in the universe of discourse $X=$ $\left\{x_{1}, x_{2}, \ldots, x_{n}\right\}$, the correlation coefficient between two INHFSs $N_{1}$ and $N_{2}$ is defined by 


$$
\begin{aligned}
& R_{I N H F S 2}\left(N_{1}, N_{2}\right)= \frac{C_{I N H F S I}\left(N_{1}, N_{2}\right)}{\max \left[C_{I N H F S I}\left(N_{1}, N_{1}\right), C_{I N H F S I}\left(N_{2}, N_{2}\right)\right]} \\
&=\frac{\left(\begin{array}{c}
\sum_{i=1}^{n}\left\{\frac{1}{l_{i}} \sum_{k=1}^{l_{i}}\left[\gamma_{1 i \sigma(k)}^{L} \cdot \gamma_{2 i \sigma(k)}^{L}+\gamma_{1 i \sigma(k)}^{U} \cdot \gamma_{2 i \sigma(k)}^{U}\right]\right. \\
+\frac{1}{p_{i}} \sum_{k=1}^{p_{i}}\left[\delta_{1 i \sigma(k)}^{L} \cdot \delta_{2 i \sigma(k)}^{L}+\delta_{1 i \sigma(k)}^{U} \cdot \delta_{2 i \sigma(k)}^{U}\right] \\
\left.+\frac{1}{q_{i}} \sum_{k=1}^{q_{i}}\left[\eta_{1 i \sigma(k)}^{L} \cdot \eta_{2 i \sigma(k)}^{L}+\eta_{1 i \sigma(k)}^{U} \cdot \eta_{2 i \sigma(k)}^{U}\right]\right\}
\end{array}\right)}{\max \left(\begin{array}{c}
\left\{\sum _ { i = 1 } ^ { n } \left\{\frac{1}{l_{i}} \sum_{k=1}^{l_{i}}\left[\left(\gamma_{1 i \sigma(k)}^{L}\right)^{2}+\left(\gamma_{1 i \sigma(k)}^{U}\right)^{2}\right]\right.\right. \\
+\frac{1}{p_{i}} \sum_{k=1}^{p_{i}}\left[\left(\delta_{1 i \sigma(k)}^{L}\right)^{2}+\left(\delta_{1 i \sigma(k)}^{U}\right)^{2}\right] \\
\left.\left.+\frac{1}{q_{i}} \sum_{k=1}^{q_{i}}\left[\left(\eta_{1 i \sigma(k)}^{L}\right)^{2}+\left(\eta_{1 i \sigma(k)}^{U}\right)^{2}\right]\right\}\right\}, \\
\left\{\sum _ { i = 1 } ^ { n } \left\{\frac{1}{l_{i}} \sum_{k=1}^{l_{i}}\left[\left(\gamma_{2 i \sigma(k)}^{L}\right)^{2}+\left(\gamma_{2 i \sigma(k)}^{U}\right)^{2}\right]\right.\right. \\
+\frac{1}{p_{i}} \sum_{k=1}^{p_{i}}\left[\left(\delta_{2 i \sigma(k)}^{L}\right)^{2}+\left(\delta_{2 i \sigma(k)}^{U}\right)^{2}\right] \\
\left.\left.+\frac{1}{q_{i}} \sum_{k=1}^{q_{i}}\left[\left(\eta_{2 i \sigma(k)}^{L}\right)^{2}+\left(\eta_{2 i \sigma(k)}^{U}\right)^{2}\right]\right\}\right\}
\end{array}\right)} .
\end{aligned}
$$

Theorem 2. The correlation coefficient $R_{I N H F S 2}\left(N_{1}, N_{2}\right)$ follows the same properties listed in Theorem 1 as follows:

(1) $R_{I N H F S 2}\left(N_{1}, N_{2}\right)=R_{I N H F S 2}\left(N_{2}, N_{1}\right)$;

(2) $0 \leqslant R_{I N H F S 2}\left(N_{1}, N_{2}\right) \leqslant 1$;

(3) $R_{I N H F S 2}\left(N_{1}, N_{2}\right)=1$, if $N_{1}=N_{2}$.

Proof. The process to prove the properties (1) and (3) is analogous to that in Theorem 1 (omitted).

(2) The inequality $R_{I N H F S 2}\left(N_{1}, N_{2}\right) \geqslant 0$ is obvious. Now, we only prove $R_{I N H F S 2}\left(N_{1}, N_{2}\right) \leqslant 1$. Based on the proof process of Theorem 1, we have

$$
C_{I N H F S I}\left(N_{1}, N_{2}\right) \leqslant\left[C_{I N H F S I}\left(N_{1}, N_{1}\right)\right]^{1 / 2}\left[C_{I N H F S I}\left(N_{2}, N_{2}\right)\right]^{1 / 2}
$$

and then

$$
C_{I N H F S I}\left(N_{1}, N_{2}\right) \leqslant \max \left[C_{I N H F S I}\left(N_{1}, N_{1}\right)\right],\left[C_{I N H F S I}\left(N_{2}, N_{2}\right)\right] .
$$

Thus, $0 \leqslant R_{I N H F S 2}\left(N_{1}, N_{2}\right) \leqslant 1$.

Especially, when $n=1$, two INHFSs $N_{1}, N_{2}$ reduce to two INHFEs $\tilde{n}_{1}=$ $\left\{\tilde{t}_{1}(x), \tilde{i}_{1}(x), \tilde{f}_{1}(x)\right\}$ and $\tilde{n}_{2}=\left\{\tilde{t}_{2}(x), \tilde{i}_{2}(x), \tilde{f}_{2}(x)\right\}$ for $x \in X$. Then, the correlation coefficients (6) and (7) reduce to a cosine similarity measure between two INHFEs and a similarity measure (or called matching function) between two INHFEs, respectively, as follows: 


$$
\begin{aligned}
S_{\text {INHFE }}\left(\tilde{n}_{1}, \tilde{n}_{2}\right)= & \frac{C_{\text {INHFE }}\left(\tilde{n}_{1}, \tilde{n}_{2}\right)}{\left[C _ { \text { INHFE } } ( \tilde { n } _ { 1 } , \tilde { n } _ { 2 } ] ^ { 1 / 2 } \left[C_{\text {INHFE }}\left(\tilde{n}_{1}, \tilde{n}_{2}\right]^{1 / 2}\right.\right.} \\
= & \left(\begin{array}{c}
\left\{\frac{1}{l} \sum_{k=1}^{l}\left[\gamma_{1 \sigma(k)}^{L} \cdot \gamma_{2 \sigma(k)}^{L}+\gamma_{1 \sigma(k)}^{U} \cdot \gamma_{2 \sigma(k)}^{U}\right]\right. \\
+\frac{1}{p} \sum_{k=1}^{p}\left[\delta_{1 \sigma(k)}^{L} \cdot \delta_{2 \sigma(k)}^{L}+\delta_{1 \sigma(k)}^{U} \cdot \delta_{2 \sigma(k)}^{U}\right] \\
\left.+\frac{1}{q} \sum_{k=1}^{q_{i}}\left[\eta_{1 \sigma(k)}^{L} \cdot \eta_{2 \sigma(k)}^{L}+\eta_{1 \sigma(k)}^{U} \cdot \eta_{2 \sigma(k)}^{U}\right]\right\}
\end{array}\right) \\
& \left(\begin{array}{c}
\left\{\frac{1}{l_{i}} \sum_{k=1}^{l_{i}}\left[\left(\gamma_{1 \sigma(k)}^{L}\right)^{2}+\left(\gamma_{1 \sigma(k)}^{U}\right)^{2}\right]\right. \\
+\frac{1}{p} \sum_{k=1}^{p}\left[\left(\delta_{1 \sigma(k)}^{L}\right)^{2}+\left(\delta_{1 \sigma(k)}^{U}\right)^{2}\right] \\
\left.+\frac{1}{q} \sum_{k=1}^{q}\left[\left(\eta_{1 \sigma(k)}^{L}\right)^{2}+\left(\eta_{1 \sigma(k)}^{U}\right)^{2}\right]\right\}^{1 / 2} \\
\times\left\{\frac{1}{l} \sum_{k=1}^{l}\left[\left(\gamma_{2 \sigma(k)}^{L}\right)^{2}+\left(\gamma_{2 \sigma(k)}^{U}\right)^{2}\right]\right. \\
+\frac{1}{p} \sum_{k=1}^{p}\left[\left(\delta_{2 \sigma(k)}^{L}\right)^{2}+\left(\delta_{2 \sigma(k)}^{U}\right)^{2}\right] \\
\left.+\frac{1}{q} \sum_{k=1}^{q_{i}}\left[\left(\eta_{2 \sigma(k)}^{L}\right)^{2}+\left(\eta_{2 \sigma(k)}^{U}\right)^{2}\right]\right\}^{1 / 2}
\end{array}\right)
\end{aligned}
$$

$$
M_{I N H F E}\left(\tilde{n}_{1}, \tilde{n}_{2}\right)=\frac{C_{I N H F E}\left(\tilde{n}_{1}, \tilde{n}_{2}\right)}{\max \left[C_{I N H F E}\left(\tilde{n}_{1}, \tilde{n}_{2}\right), C_{I N H F E}\left(\tilde{n}_{1}, \tilde{n}_{2}\right)\right]}
$$

$$
=\frac{\left(\begin{array}{c}
\left\{\frac{1}{l_{i}} \sum_{k=1}^{l_{i}}\left[\gamma_{1 \sigma(k)}^{L} \cdot \gamma_{2 \sigma(k)}^{L}+\gamma_{1 \sigma(k)}^{U} \cdot \gamma_{2 \sigma(k)}^{U}\right]\right. \\
+\frac{1}{p} \sum_{k=1}^{p}\left[\delta_{1 i \sigma(k)}^{L} \cdot \delta_{2 \sigma(k)}^{L}+\delta_{1 \sigma(k)}^{U} \cdot \delta_{2 \sigma(k)}^{U}\right] \\
\left.+\frac{1}{q} \sum_{k=1}^{q}\left[\eta_{1 \sigma(k)}^{L} \cdot \eta_{2 \sigma(k)}^{L}+\eta_{1 \sigma(k)}^{U} \cdot \eta_{2 \sigma(k)}^{U}\right]\right\}
\end{array}\right)}{\max \left(\begin{array}{c}
\left\{\frac{1}{l} \sum_{k=1}^{l}\left[\left(\gamma_{1 \sigma(k)}^{L}\right)^{2}+\left(\gamma_{1 \sigma(k)}^{U}\right)^{2}\right]\right. \\
+\frac{1}{p} \sum_{k=1}^{p}\left[\left(\delta_{1 \sigma(k)}^{L}\right)^{2}+\left(\delta_{1 \sigma(k)}^{U}\right)^{2}\right] \\
\left.+\frac{1}{q} \sum_{k=1}^{q}\left[\left(\eta_{1 \sigma(k)}^{L}\right)^{2}+\left(\eta_{1 \sigma(k)}^{U}\right)^{2}\right]\right\}, \\
\left\{\begin{array}{c}
1 \\
l
\end{array} \sum_{k=1}^{l}\left[\left(\gamma_{2 \sigma(k)}^{L}\right)^{2}+\left(\gamma_{2 \sigma(k)}^{U}\right)^{2}\right]\right. \\
+\frac{1}{p} \sum_{k=1}^{p}\left[\left(\delta_{2 \sigma(k)}^{L}\right)^{2}+\left(\delta_{2 \sigma(k)}^{U}\right)^{2}\right] \\
\left.+\frac{1}{q} \sum_{k=1}^{q}\left[\left(\eta_{2 \sigma(k)}^{L}\right)^{2}+\left(\eta_{2 \sigma(k)}^{U}\right)^{2}\right]\right\}
\end{array}\right)}
$$

where $\tilde{\gamma}_{1 \sigma(k)}=\left[\gamma_{1 \sigma(k)}^{L}, \gamma_{1 \sigma(k)}^{U}\right] \in \tilde{t}_{1}(x)$ and $\tilde{\gamma}_{2 \sigma(k)}=\left[\gamma_{2 \sigma(k)}^{L}, \gamma_{2 \sigma(k)}^{U}\right] \in \tilde{t}_{2}(x)$ for $k=$ $1,2, \ldots, l, \tilde{\delta}_{1 \sigma(k)}=\left[\delta_{1 \sigma(k)}^{L}, \delta_{1 \sigma(k)}^{U}\right] \in \tilde{i}_{1}(x)$ and $\tilde{\delta}_{2 \sigma(k)}=\left[\delta_{2 \sigma(k)}^{L}, \delta_{2 \sigma(k)}^{U}\right] \in \tilde{i}_{2}(x)$ for $k=$ $1,2, \ldots, p$, and $\tilde{\eta}_{1 \eta(k)}=\left[\eta_{1 \sigma(k)}^{L}, \eta_{1 \sigma(k)}^{U}\right] \in \tilde{f}_{1}(x)$ and $\tilde{\eta}_{2 \eta(k)}=\left[\eta_{2 \sigma(k)}^{L}, \eta_{2 \sigma(k)}^{U}\right] \in \tilde{f}_{2}(x)$ for $k=1,2, \ldots, q$, and $l, p, q$ are the number of intervals in $\tilde{t}_{1}(x)$ and $\tilde{t}_{2}(x), \tilde{i}_{1}(x)$ and $\tilde{i}_{2}(x)$, $\tilde{f}_{1}(x)$ and $\tilde{f}_{2}(x)$, respectively.

Therefore, Eqs. (8) and (9) are special cases of Eqs. (6) and (7).

However, the differences of importance are considered in the elements in the universe. Therefore, we need to take the weights of the elements $x_{i}(i=1,2, \ldots, n)$ into account. In the following, we develop two weighted correlation coefficients between INHFSs.

Let $w_{i}$ be the weight for each element $x_{i}(i=1,2, \ldots, n), w_{i} \in[0,1]$, and $\sum_{i=1}^{n} w_{i}=1$, then we have two weighted correlation coefficients between the INHFSs 
$A$ and $B$, respectively, as follows:

$$
\begin{aligned}
& R_{I N H F S 3}\left(N_{1}, N_{2}\right)=\frac{C_{I N H F S 2}\left(N_{1}, N_{2}\right)}{\left[C_{I N H F S 2}\left(N_{1}, N_{1}\right)\right]^{1 / 2}\left[C_{I N H F S 2}\left(N_{2}, N_{2}\right)\right]^{1 / 2}} \\
& =\frac{\left(\begin{array}{c}
\sum_{i=1}^{n} w_{i}\left\{\frac{1}{l_{i}} \sum_{k=1}^{l_{i}}\left[\gamma_{1 i \sigma(k)}^{L} \cdot \gamma_{2 i \sigma(k)}^{L}+\gamma_{1 i \sigma(k)}^{U} \cdot \gamma_{2 i \sigma(k)}^{U}\right]\right. \\
+\frac{1}{p_{i}} \sum_{k=1}^{p_{i}}\left[\delta_{1 i \sigma(k)}^{L} \cdot \delta_{2 i \sigma(k)}^{L}+\delta_{1 i \sigma(k)}^{U} \cdot \delta_{2 i \sigma(k)}^{U}\right] \\
\left.+\frac{1}{q_{i}} \sum_{k=1}^{q_{i}}\left[\eta_{1 i \sigma(k)}^{L} \cdot \eta_{2 i \sigma(k)}^{L}+\eta_{1 i \sigma(k)}^{U} \cdot \eta_{2 i \sigma(k)}^{U}\right]\right\}
\end{array}\right)}{\left(\begin{array}{c}
\left\{\sum _ { i = 1 } ^ { n } w _ { i } \left\{\frac{1}{l_{i}} \sum_{k=1}^{l_{i}}\left[\left(\gamma_{1 i \sigma(k)}^{L}\right)^{2}+\left(\gamma_{1 i \sigma(k)}^{U}\right)^{2}\right]\right.\right. \\
\quad+\frac{1}{p_{i}} \sum_{k=1}^{p_{i}}\left[\left(\delta_{1 i \sigma(k)}^{L}\right)^{2}+\left(\delta_{1 i \sigma(k)}^{U}\right)^{2}\right] \\
\left.\left.\quad+\frac{1}{q_{i}} \sum_{k=1}^{q_{i}}\left[\left(\eta_{1 i \sigma(k)}^{L}\right)^{2}+\left(\eta_{1 i \sigma(k)}^{U}\right)^{2}\right]\right\}\right\}^{1 / 2} \\
\quad \times\left\{\sum _ { i = 1 } ^ { n } w _ { i } \left\{\frac{1}{l_{i}} \sum_{k=1}^{l_{i}}\left[\left(\gamma_{2 i \sigma(k)}^{L}\right)^{2}+\left(\gamma_{2 i \sigma(k)}^{U}\right)^{2}\right]\right.\right. \\
\quad+\frac{1}{p_{i}} \sum_{k=1}^{p_{i}}\left[\left(\delta_{2 i \sigma(k)}^{L}\right)^{2}+\left(\delta_{2 i \sigma(k)}^{U}\right)^{2}\right] \\
\left.\left.\quad+\frac{1}{q_{i}} \sum_{k=1}^{q_{i}}\left[\left(\eta_{2 i \sigma(k)}^{L}\right)^{2}+\left(\eta_{2 i \sigma(k)}^{U}\right)^{2}\right]\right\}\right\}^{1 / 2}
\end{array}\right)}
\end{aligned}
$$

$$
\begin{aligned}
& R_{I N H F S 4}\left(N_{1}, N_{2}\right)= \frac{C_{I N H F S 2}\left(N_{1}, N_{2}\right)}{\max \left[C_{I N H F S 2}\left(N_{1}, N_{1}\right), C_{I N H F S 2}\left(N_{2}, N_{2}\right)\right]} \\
&=\frac{\left(\begin{array}{c}
\sum_{i=1}^{n} w_{i}\left\{\frac{1}{l_{i}} \sum_{k=1}^{l_{i}}\left[\gamma_{1 i \sigma(k)}^{L} \cdot \gamma_{2 i \sigma(k)}^{L}+\gamma_{1 i \sigma(k)}^{U} \cdot \gamma_{2 i \sigma(k)}^{U}\right]\right. \\
+\frac{1}{p_{i}} \sum_{k=1}^{p_{i}}\left[\delta_{1 i \sigma(k)}^{L} \cdot \delta_{2 i \sigma(k)}^{L}+\delta_{1 i \sigma(k)}^{U} \cdot \delta_{2 i \sigma(k)}^{U}\right] \\
\left.+\frac{1}{q_{i}} \sum_{k=1}^{q_{i}}\left[\eta_{1 i \sigma(k)}^{L} \cdot \eta_{2 i \sigma(k)}^{L}+\eta_{1 i \sigma(k)}^{U} \cdot \eta_{2 i \sigma(k)}^{U}\right]\right\}
\end{array}\right)}{\max \left(\begin{array}{c}
\left\{\sum _ { i = 1 } ^ { n } w _ { i } \left\{\frac{1}{l_{i}} \sum_{k=1}^{l_{i}}\left[\left(\gamma_{1 i \sigma(k)}^{L}\right)^{2}+\left(\gamma_{1 i \sigma(k)}^{U}\right)^{2}\right]\right.\right. \\
+\frac{1}{p_{i}} \sum_{k=1}^{p_{i}}\left[\left(\delta_{1 i \sigma(k)}^{L}\right)^{2}+\left(\delta_{1 i \sigma(k)}^{U}\right)^{2}\right] \\
\left.\left.+\frac{1}{q_{i}} \sum_{k=1}^{q_{i}}\left[\left(\eta_{1 i \sigma(k)}^{L}\right)^{2}+\left(\eta_{1 i \sigma(k)}^{U}\right)^{2}\right]\right\}\right\}, \\
\left\{\sum _ { i = 1 } ^ { n } w _ { i } \left\{\frac{1}{l_{i}} \sum_{k=1}^{l_{i}}\left[\left(\gamma_{2 i \sigma(k)}^{L}\right)^{2}+\left(\gamma_{2 i \sigma(k)}^{U}\right)^{2}\right]\right.\right. \\
+\frac{1}{p_{i}} \sum_{k=1}^{p_{i}}\left[\left(\delta_{2 i \sigma(k)}^{L}\right)^{2}+\left(\delta_{2 i \sigma(k)}^{U}\right)^{2}\right] \\
\left.\left.+\frac{1}{q_{i}} \sum_{k=1}^{q_{i}}\left[\left(\eta_{2 i \sigma(k)}^{L}\right)^{2}+\left(\eta_{2 i \sigma(k)}^{U}\right)^{2}\right]\right\}\right\}
\end{array}\right)} .
\end{aligned}
$$

If $w=(1 / n, 1 / n, \ldots, 1 / n)^{T}$, then Eqs. (10) and (11) reduce to Eqs. (6) and (7), respectively. Note that both $R_{I N H F S 3}(A, B)$ and $R_{I N H F S 4}(A, B)$ also satisfy the three properties of Theorem 1 .

Theorem 3. Let $w_{i}$ be the weight for each element $x_{i}(i=1,2, \ldots, n), w_{i} \in[0,1]$, and $\sum_{i=1}^{n} w_{i}=1$, then the weighted correlation coefficient $R_{I N H F S 3}\left(N_{1}, N_{2}\right)$ defined in Eq. (10) satisfies the following properties:

(1) $R_{I N H F S 3}\left(N_{1}, N_{2}\right)=R_{I N H F S 3}\left(N_{2}, N_{1}\right)$; 
(2) $0 \leqslant R_{I N H F S 3}\left(N_{1}, N_{2}\right) \leqslant 1$;

(3) $R_{I N H F S 3}\left(N_{1}, N_{2}\right)=1$, if $N_{1}=N_{2}$.

Since the process to prove these properties is similar to that in Theorem 1, we do not repeat it here.

Theorem 4. Let $w_{i}$ be the weight for each element $x_{i}(i=1,2, \ldots, n), w_{i} \in[0,1]$, and $\sum_{i=1}^{n} w_{i}=1$, then the weighted correlation coefficient $R_{I N H F S 4}\left(N_{1}, N_{2}\right)$ defined in Eq. (11) satisfies the following properties:

(1) $R_{\text {INHFS4 }}\left(N_{1}, N_{2}\right)=R_{I N H F S 4}\left(N_{2}, N_{1}\right)$;

(2) $0 \leqslant R_{I N H F S 4}\left(N_{1}, N_{2}\right) \leqslant 1$;

(3) $R_{\text {INHFS4 }}\left(N_{1}, N_{2}\right)=1$, if $N_{1}=N_{2}$.

Since the process to prove these properties is similar to that in Theorem 2, we do not repeat it here.

\section{Decision-Making Method Based on the Correlation Coefficients}

In this section, we propose a multiple attribute decision-making method based on the weighted correlation coefficients between INHFSs under interval neutrosophic hesitant fuzzy environment.

Let $A=\left\{A_{1}, A_{2}, \ldots, A_{m}\right\}$ be a set of alternatives and $C=\left\{C_{1}, C_{2}, \ldots, C_{n}\right\}$ be a set of attributes. Assume that the weight of an attribute $C_{j}(j=1,2, \ldots, n)$, entered by the decision-maker, is $w_{j}, w_{j} \in[0,1]$ and $\sum_{j=1}^{n} w_{j}=1$. In the evaluation of the alternatives, the characteristic of an alternative $A_{i}(i=1,2, \ldots, m)$ on an attribute $C_{j}(j=1,2, \ldots, n)$ is represented by an INHFS:

$$
A_{i}=\left\{\left\langle C_{j}, \tilde{t}_{i}\left(C_{j}\right), \tilde{i}_{i}\left(C_{j}\right), \tilde{f}_{i}\left(C_{j}\right)\right\rangle \mid C_{j} \in C, j=1,2, \ldots, n\right\},
$$

where $\tilde{t}_{i}\left(C_{j}\right)=\left\{\tilde{\gamma} \mid \tilde{\gamma} \in \tilde{t}_{i}\left(C_{j}\right)\right\}, \tilde{i}_{i}\left(C_{j}\right)=\left\{\tilde{\delta} \mid \tilde{\delta} \in \tilde{i}_{i}\left(C_{j}\right)\right\}$, and $\tilde{f}_{i}\left(C_{j}\right)=\left\{\tilde{\eta} \mid \tilde{\eta} \in \tilde{f}_{i}\left(C_{j}\right)\right\}$ are three sets of some interval values in real unit interval $[0,1]$, denoting the possible truth-membership hesitant degrees, indeterminacy-membership hesitant degrees, and falsity-membership hesitant degrees of the element $C_{j} \in C$ to the set $A_{i}$, respectively. When the decision makers are required to evaluate the alternative $A_{i}(i=1,2, \ldots, m)$ under the attribute $C_{j}(j=1,2, \ldots, n)$, they may assign a set of several possible interval values to each of truth-membership degrees, indeterminacy-membership degrees, and falsity-membership degrees to which an alternative $A_{i}(i=1,2, \ldots, m)$ satisfies or unsatisfies or indeterminates an attribute $C_{j}(j=1,2, \ldots, n)$, and then these evaluated values can be expressed as an INHFE $\tilde{n}_{i}\left(C_{j}\right)=\left\{\tilde{t}_{i}\left(C_{j}\right), \tilde{i}_{i}\left(C_{j}\right), \tilde{f}_{i}\left(C_{j}\right)\right\}$ in an INHFS $A_{i}(i=1,2, \ldots, m ; j=1,2, \ldots, n)$. For convenience, the INHFE $\tilde{n}_{i}\left(C_{j}\right)=$ $\left\{\tilde{t}_{i}\left(C_{j}\right), \tilde{i}_{i}\left(C_{j}\right), \tilde{f}_{i}\left(C_{j}\right)\right\}$ is denoted by the simplified symbol $\tilde{n}_{i j}=\left\{\tilde{t}_{i j}, \tilde{i}_{i j}, \tilde{f}_{i j}\right\} \quad(i=$ $1,2, \ldots, m ; j=1,2, \ldots, n)$. Thus, we can elicit a interval neutrosophic hesitant fuzzy decision matrix $D=\left(\tilde{n}_{i j}\right)_{m \times n}$. 
The weight vector of attributes for the different importance of each attribute is given as $w=\left(w_{1}, w_{2}, \ldots, w_{n}\right)^{T}$, where $w_{j} \geqslant 0, j=1,2, \ldots, n$, and $\sum_{j=1}^{n} w_{j}=1$.

In multiple attribute decision making problems, the concept of ideal point has been used to help identify the best alternative in the decision set. Although the ideal alternative does not exist in real world, it does provide a useful theoretical construct against which to evaluate alternatives $(\mathrm{Ye}, 2013)$. Therefore, we can define an ideal INHFE as $\tilde{n}_{i j}^{*}=$ $\{\{[1,1]\},\{[0,0]\},\{[0,0]\}\}$ in the ideal alternative $A^{*}$. In the proposed decision-method we can utilize either Eq. (10) or Eq. (11) as the final decision. By applying Eq. (10) the weighted correlation coefficient between an alternative $A_{i}(i=1,2, \ldots, m)$ and the ideal alternative $A^{*}$ is given by

$$
\begin{aligned}
& R_{\text {INHFSS }}\left(A_{i}, A^{*}\right) \\
& =\frac{\sum_{j=1}^{n} w_{j}\left[\frac{1}{l_{i j}} \sum_{k=1}^{l_{i j}}\left(\gamma_{i j \sigma(k)}^{L}+\gamma_{i j \sigma(k)}^{U}\right)\right]}{\left(2 \sum_{j=1}^{n} w_{j}\left\{\begin{array}{c}
\frac{1}{l_{i j}} \sum_{k=1}^{l_{i j}}\left[\left(\gamma_{i j \sigma(k)}^{L}\right)^{2}+\left(\gamma_{i j \sigma(k)}^{U}\right)^{2}\right] \\
+\frac{1}{p_{i j}} \sum_{k=1}^{p_{i j}}\left[\left(\delta_{i j \sigma(k)}^{L}\right)^{2}+\left(\delta_{i j \sigma(k)}^{U}\right)^{2}\right] \\
+\frac{1}{q_{i j}} \sum_{k=1}^{q_{i j}}\left[\left(\eta_{i j \sigma(k)}^{L}\right)^{2}+\left(\eta_{i j \sigma(k)}^{U}\right)^{2}\right]
\end{array}\right]\right)^{1 / 2}}
\end{aligned}
$$

where $\tilde{\gamma}_{i j}=\left[\gamma_{i j}^{L}, \gamma_{i j}^{U}\right] \in \tilde{t}_{i j}, \tilde{\delta}_{i j}=\left[\delta_{i j}^{L}, \delta_{i j}^{U}\right] \in \tilde{i}_{i j}, \tilde{\eta}_{i j}=\left[\eta_{i j}^{L}, \eta_{i j}^{U}\right] \in \tilde{f}_{i j}, l_{i j}, p_{i j}$, and $q_{i j}$ are the numbers of interval elements in $\tilde{t}_{i j}, \tilde{i}_{i j}, \tilde{f}_{i j}$, respectively, and $R_{I N H F S S}\left(A_{i}, A^{*}\right) \in[0,1]$ for $i=1,2, \ldots, n$.

Or by applying Eq. (11), the weighted correlation coefficient between an alternative $A_{i}(i=1,2, \ldots, m)$ and the ideal alternative $A^{*}$ is given by

$$
\begin{aligned}
& R_{I N H F S 6}\left(A_{i}, A^{*}\right) \\
& \max \left(2, \sum_{j=1}^{n} w_{j}\left\{\begin{array}{c}
\frac{1}{l_{i j}} \sum_{k=1}^{l_{i j}}\left[\left(\gamma_{i j \sigma(k)}^{L}\right)^{2}+\left(\gamma_{i j \sigma(k)}^{U}\right)^{2}\right] \\
+\frac{1}{p_{i j}} \sum_{k=1}^{p_{i j}}\left[\left(\delta_{i j \sigma(k)}^{L}\right)^{2}+\left(\delta_{i j \sigma(k)}^{U}\right)^{2}\right] \\
+\frac{1}{q_{i j}} \sum_{k=1}^{q_{i j}}\left[\left(\eta_{i j \sigma(k)}^{L}\right)^{2}+\left(\eta_{i j \sigma(k)}^{U}\right)^{2}\right]
\end{array}\right\}\right) .
\end{aligned}
$$

Through the correlation coefficient $R_{I N H F S k}\left(A_{i}, A^{*}\right)(k=5$ or $6 ; i=1,2, \ldots, m)$, we can obtain the ranking order of all alternatives and the best one(s).

\section{Illustrative Example}

In this section, an illustrative example for the multiple attribute decision-making problem of investment alternatives is given to demonstrate the application and effectiveness of the proposed decision-making method.

Let us consider the decision-making problem adapted from (Ye, 2013). There is an investment company, which wants to invest a sum of money in the best option. There 
Table 1

Interval neutrosophic hesitant fuzzy decision matrix $D$.

\begin{tabular}{llll}
\hline & $C_{1}$ & $C_{2}$ & $C_{3}$ \\
\hline$A_{1}$ & $\{\{[0.3,0.4],[0.4,0.4]$, & $\{\{[0.4,0.5],[0.5,0.6]\}$, & $\{\{[0.2,0.3]\},\{[0.1,0.2]\}$, \\
& $[0.4,0.5]\},\{[0.1,0.2]\}$, & $\{[0.2,0.3]\},\{[0.2,0.3]$, & $\{[0.4,0.5],[0.5,0.6]\}\}$ \\
& $\{[0.3,0.4]\}$ & $[0.3,0.4]\}\}$ & \\
$A_{2}$ & $\{\{[0.6,0.7]\},\{[0.1,0.2]\}$ & $\{\{[0.6,0.7]\},\{[0.1,0.1]\}$, & $\{\{[0.6,0.7]\},\{[0.1,0.2]\}$, \\
& $\{[0.1,0.2],[0.2,0.3]\}\}$ & $\{\{[0.2,0.3]\}\}$ & $\{[0.1,0.2]\}\}$ \\
$A_{3}$ & $\{\{[0.3,0.4],[0.5,0.6]\}$, & $\{\{[0.5,0.6]\},\{[0.2,0.3]\}$, & $\{\{[0.5,0.6]\},\{[0.1,0.2]$, \\
& $\{[0.2,0.4]\},\{[0.2,0.3]\}\}$ & $\{[0.3,0.4]\}\}$ & $[0.2,0.3]\},\{[0.2,0.3]\}\}$ \\
$A_{4}$ & $\{\{[0.7,0.8]\},\{[0,0.1]\}$ & $\{\{[0.6,0.7]\},\{[0,0.1]\}$, & $\{\{[0.3,0.5]\},\{[0.2,0.3]\}$, \\
& $\{[0.1,0.2]\}\}$ & $\{[0.2,0.2]\}\}$ & $\{[0.1,0.2],[0.3,0.3]\}\}$ \\
\hline
\end{tabular}

is a panel with four possible alternatives to invest the money: (1) $A_{1}$ is a car company; (2) $A_{2}$ is a food company; (3) $A_{3}$ is a computer company; (4) $A_{4}$ is an arms company. The investment company must take a decision according to the three attributes: (1) $C_{1}$ is the risk; (2) $C_{2}$ is the growth; (3) $C_{3}$ is the environmental impact. The weight vector of the attributes is given by $w=(0.35,0.25,0.4)^{T}(\mathrm{Ye}, 2013)$. The four possible alternatives are to be evaluated under the above three attributes by the form of INHFEs, and then we can obtain the interval neutrosophic hesitant fuzzy decision matrix $D$, as shown in Table 1 .

Then, we utilize the developed approach to obtain the ranking order of the alternatives and the most desirable one(s).

By using Eq. (12), we can obtain the values of the correlation coefficient $R_{\text {INHFS5 }}\left(A_{i}, A^{*}\right)(i=1,2,3,4)$. Take an alternative $A_{1}$ as an example, we have

$$
\begin{aligned}
& R_{I N H F S 5}\left(A_{1}, A^{*}\right) \\
& =\frac{\sum_{j=1}^{3} w_{j}\left[\frac{1}{l_{1 j}} \sum_{k=1}^{l_{1 j}}\left(\gamma_{1 j \sigma(k)}^{L}+\gamma_{1 j \sigma(k)}^{U}\right)\right]}{\left.2 \sum_{j=1}^{3} w_{j}\left\{\begin{array}{c}
\frac{1}{l_{1 j}} \sum_{k=1}^{l_{1 j}}\left[\left(\gamma_{i j \sigma(k)}^{L}\right)^{2}+\left(\gamma_{1 j \sigma(k)}^{U}\right)^{2}\right] \\
+\frac{1}{p_{1 j}} \sum_{k=1}^{p_{1 j}}\left[\left(\delta_{1 j \sigma(k)}^{L}\right)^{2}+\left(\delta_{1 j \sigma(k)}^{U}\right)^{2}\right] \\
+\frac{1}{q_{1 j}} \sum_{k=1}^{q_{1 j}}\left[\left(\eta_{1 j \sigma(k)}^{L}\right)^{2}+\left(\eta_{1 j \sigma(k)}^{U}\right)^{2}\right]
\end{array}\right\}\right)} \\
& 0.35(0.3+0.4+0.4+0.4+0.4+0.5) / 3 \\
& =\frac{+0.25(0.4+0.5+0.5+0.6) / 2+0.4(0.2+0.3)}{\left.2\left\{\begin{array}{l}
0.35\left[\left(0.3^{2}+0.4^{2}+0.4^{2}+0.4^{2}+0.4^{2}+0.5^{2}\right) / 3\right. \\
\left.+\left(0.1^{2}+0.2^{2}\right)+\left(0.3^{2}+0.4^{2}\right)\right] \\
+0.25\left[\left(0.4^{2}+0.5^{2}+0.5^{2}+0.6^{2}\right) / 2\right. \\
\left.+\left(0.2^{2}+0.3^{2}\right)+\left(0.2^{2}+0.3^{2}+0.3^{2}+0.4^{2}\right) / 2\right] \\
+0.4\left[\left(0.2^{2}+0.3^{2}\right)+\left(0.1^{2}+0.2^{2}\right)\right. \\
\left.+\left(0.4^{2}+0.5^{2}+0.5^{2}+0.6^{2}\right) / 2\right]
\end{array}\right\}\right)} \\
& =0.6157 \text {. }
\end{aligned}
$$


Similar to the above calculation, we can obtain the following values of the correlation coefficient $R_{I N H F S 5}\left(A_{i}, A^{*}\right)(i=2,3,4)$ :

$$
\begin{aligned}
& R_{I N H F S 5}\left(A_{2}, A^{*}\right)=0.9302, \quad R_{I N H F S 5}\left(A_{3}, A^{*}\right)=0.7921, \quad \text { and } \\
& R_{I N H F S S}\left(A_{4}, A^{*}\right)=0.8792 .
\end{aligned}
$$

Thus, the ranking order of the four alternatives is $A_{2} \succ A_{4} \succ A_{3} \succ A_{1}$. Therefore, the alternative $A_{2}$ is the best choice among the four alternatives.

Or by using Eq. (13), we can also obtain the values of the correlation coefficient $R_{I N H F S 6}\left(A_{i}, A^{*}\right)(i=1,2,3,4)$. Take an alternative $A_{1}$ as an example, we have

$$
\begin{aligned}
& R_{I N H F S \sigma}\left(A_{1}, A^{*}\right) \\
& =\frac{\sum_{j=1}^{3} w_{j}\left[\frac{1}{l_{1 j}} \sum_{k=1}^{l_{1 j}}\left(\gamma_{1 j \sigma(k)}^{L}+\gamma_{1 j \sigma(k)}^{U}\right)\right]}{\max \left(2, \sum_{j=1}^{3} w_{j}\left\{\begin{array}{c}
\frac{1}{l_{1 j}} \sum_{k=1}^{l_{1 j}}\left[\left(\gamma_{1 j \sigma(k)}^{L}\right)^{2}+\left(\gamma_{1 j \sigma(k)}^{U}\right)^{2}\right] \\
+\frac{1}{p_{1 j}} \sum_{k=1}^{p_{1 j}}\left[\left(\delta_{1 j \sigma(k)}^{L}\right)^{2}+\left(\delta_{1 j \sigma(k)}^{U}\right)^{2}\right] \\
+\frac{1}{q_{1 j}} \sum_{k=1}^{q_{1 j}}\left[\left(\eta_{1 j \sigma(k)}^{L}\right)^{2}+\left(\eta_{1 j \sigma(k)}^{U}\right)^{2}\right]
\end{array}\right\}\right)} \\
& 0.35(0.3+0.4+0.4+0.4+0.4+0.5) / 3 \\
& =\frac{+0.25(0.4+0.5+0.5+0.6) / 2+0.4(0.2+0.3)}{\max \left(2,\left\{\begin{array}{l}
0.35\left[\left(0.3^{2}+0.4^{2}+0.4^{2}+0.4^{2}+0.4^{2}+0.5^{2}\right) / 3\right. \\
\left.+\left(0.1^{2}+0.2^{2}\right)+\left(0.3^{2}+0.4^{2}\right)\right] \\
+0.25\left[\left(0.4^{2}+0.5^{2}+0.5^{2}+0.6^{2}\right) / 2\right. \\
\left.+\left(0.2^{2}+0.3^{2}\right)+\left(0.2^{2}+0.3^{2}+0.3^{2}+0.4^{2}\right) / 2\right] \\
+0.4\left[\left(0.2^{2}+0.3^{2}\right)+\left(0.1^{2}+0.2^{2}\right)\right. \\
\left.+\left(0.4^{2}+0.5^{2}+0.5^{2}+0.6^{2}\right) / 2\right]
\end{array}\right\}\right)} \\
& =0.365 \text {. }
\end{aligned}
$$

Similar to the above calculation, we can obtain the following values of the correlation coefficient $R_{I N H F S 6}\left(A_{i}, A^{*}\right)(i=2,3,4)$ :

$$
\begin{aligned}
& R_{\text {INHFS }}\left(A_{2}, A^{*}\right)=0.65, \quad R_{\text {INHFS }}\left(A_{3}, A^{*}\right)=0.515, \quad \text { and } \\
& R_{\text {INHFS6 }}\left(A_{4}, A^{*}\right)=0.585 .
\end{aligned}
$$

Therefore, the ranking order of the four alternatives is $A_{2} \succ A_{4} \succ A_{3} \succ A_{1}$. Obviously, the alternative $A_{2}$ is also the best choice among the four alternatives.

From the above results we can see that the above two kinds of ranking orders of the four alternatives and the best choice are the same with respect to using different correlation coefficients, which are in agreement with the results of Ye's method (Ye 2013). The above example indicates that the proposed decision-making method is applicable and effective under interval neutrosophic hesitant fuzzy environment. As mentioned above, INHFSs are the extension of the existing sets such as HFSs, IVHFSs, 
DHFSs, SVNSs, INSs, which are special cases of the INHFSs. Therefore, the correlation coefficients of INHFSs proposed in this paper are the further extension of the existing correlation coefficients (Gerstenkorn and Manko, 1991; Bustince and Burillo, 1995; Hong, 1998; Chiang and Lin, 1999; Wang and Li, 1999; Hung and Wu, 2002; Hong, 2006; Park et al., 2009; Szmidt and Kacprzyk, 2010; Ye, 2010; Wei et al., 2011; Chen et al., 2013a; Ye, 2013, 2014a). On the one hand, compared with the decision making methods based on the correlation coefficients (Park et al., 2009; Ye, 2010; Wei et al., 2011; Ye, 2013, 2014a), the decision-making method based on the correlation coefficients of INHFSs further extend the existing decision making methods (Park et al., 2009; Wei et al., 2011; Ye, 2010, 2013, 2014a) since the later is special cases of the former. Therefore, the proposed interval neutrosophic hesitant fuzzy decision-making method is more general and more practical than existing decision-making methods (Park et al., 2009; Wei et al., 2011; Ye, 2010, 2013, 2014a). On the other hand, compared with the decision making methods based on the aggregation operators (Xia and Xu, 2011; Ye, 2014d), our decision making method based on the correlation coefficients can avoid complex information aggregation and can directly use the derived correlation coefficient formula to rank the alternatives. Therefore, the decision making approach in this paper is more simple and more convenient than the decision making methods in Xia and Xu (2011), Ye (2014d). Thus, the comparisons clearly demonstrate the benefits of the proposed decision-making approach based on correlation coefficients of INHFSs. Furthermore, in the proposed decision making method, the decision makers can select any one of two correlation coefficients of INHFSs according to their preference to obtain the final decision.

\section{Conclusions}

This paper proposed the concept of INHFSs and their basic operations. Then, we developed some correlation coefficients between INHFSs as a further generalization of the correlation coefficients for SVNSs and IVHFSs and investigated their properties and the relation between some similarity measures and the correlation coefficients for INHFSs. Furthermore, a multiple attribute decision-making method based on the correlation coefficients of INHFSs has been established under interval neutrosophic hesitant fuzzy environment. Through the correlation coefficients between each alternative and the ideal alternative, we can obtain the ranking order of all alternatives and the best one. Finally, an illustrative example demonstrated the application and effectiveness of the developed decision-making approach. The proposed interval neutrosophic hesitant fuzzy decisionmaking method is more suitable for decision making problems with the incomplete, indeterminate, and inconsistent information and more general and more practical than existing decision-making approaches. Therefore, the techniques proposed in this paper extend existing correlation coefficients and decision-making methods and can provide a new way for decision-makers. In the future, we shall apply the correlation coefficients between INHFSs to other domains, such as expert system, clustering analysis, and medical diagnosis. 


\section{References}

Bonizzoni, P., Vedova, G.D., Dondi, R., Jiang, T. (2008). Correlation clustering and consensus clustering. Lecture Notes in Computer Science, 3827, 226-235.

Broumi, S. Smarandache, F. (2013). Correlation coefficient of interval neutrosophic set. Applied Mechanics and Materials, 436, 511-517.

Bustince, H., Burillo, P. (1995). Correlation of interval-valued intuitionistic fuzzy sets. Fuzzy Sets and Systems, 74, 237-244.

Chen, N., Xu, Z.S., Xia, M.M. (2013a). Correlation coefficients of hesitant fuzzy sets and their applications to clustering analysis. Applied Mathematical Modelling, 37, 2197-2211.

Chen, N., Xu, Z.S., Xia, M.M. (2013b). Interval-valued hesitant preference relations and their applications to group decision making. Knowledge-Based Systems, 37, 528-540.

Chiang, D.A., Lin, N.P. (1999). Correlation of fuzzy sets. Fuzzy Sets and Systems, 102, 221-226.

Gerstenkorn, T., Manko, J. (1991). Correlation of intuitionistic fuzzy sets. Fuzzy Sets and Systems, 44, $39-43$.

Hong, D.H. (1998). A note on correlation of interval-valued intuitionistic fuzzy sets. Fuzzy Sets and Systems, 95, 113-117.

Hong, D.H. (2006). Fuzzy measures for a correlation coefficient of fuzzy numbers under Tw (the weakest t-norm)-based fuzzy arithmetic operations. Information Sciences, 176, 150-160.

Hung, W.L., Wu, J.W. (2002). Correlation of intuitionistic fuzzy sets by centroid method. Information Sciences, 144, 219-225.

Kriegel, H.P., Kroger, P., Schubert, E., Zimek, A. (2008). A general framework for increasing the robustness of PCA-based correlation clustering algorithms. Lecture Notes in Computer Science, 5069, 418-435.

Liu, P.D. (2013). Some generalized dependent aggregation operators with intuitionistic linguistic numbers and their application to group decision making. Journal of Computer and System Sciences, 79(1), 131-143.

Liu, P.D., Jin, F. (2012). Methods for aggregating intuitionistic uncertain linguistic variables and their application to group decision making. Information Sciences, 205, 58-71.

Liu, P.D., Wang, Y.M. (2014). Multiple attribute group decision making methods based on intuitionistic linguistic power generalized aggregation operators. Applied Soft Computing, 17, 90-104.

Liu, P.D., Liu, Z.M., Zhang, X. (2014). Some intuitionistic uncertain linguistic Heronian mean operators and their application to group decision making. Applied Mathematics and Computation, 230, 570-586.

Park, D.G., Kwun, Y.C., Park, J.H., Park, I.Y. (2009). Correlation coefficient of interval-valued intuitionistic fuzzy sets and its application to multiple attribute group decision making problems. Mathematical and Computer Modelling, 50, 1279-1293.

Smarandache, F. (1999). A Unifying Field in Logics. Neutrosophy: Neutrosophic Probability, Set and Logic. American Research Press, Rehoboth

Szmidt, E., Kacprzyk, J. (2010). Correlation of intuitionistic fuzzy sets. Lecture Notes in Computer Science, $6178,169-177$.

Torra, V. (2010). Hesitant fuzzy sets. International Journal of Intelligent Systems, 25, 529-539.

Torra, V., Narukawa, Y. (2009). On hesitant fuzzy sets and decision. In: The 18th IEEE International Conference on Fuzzy Systems, Jeju Island, Korea, pp. 1378-1382.

Wang, G.J., Li, X.P. (1999). Correlation and information energy of interval-valued fuzzy numbers. Fuzzy Sets and Systems, 103, 169-175.

Wang, H., Smarandache, F., Zhang, Y.Q., Sunderraman, R. (2005). Interval Neutrosophic Sets and Logic: Theory and Applications in Computing. Hexis, Phoenix.

Wang, H., Smarandache, F., Zhang, Y.Q., Sunderraman, R. (2010). Single valued neutrosophic sets. Multispace and Multistructure, 4, 410-413.

Wei, G.W., Wang, H.J., Lin, R. (2011). Application of correlation coefficient to interval-valued intuitionistic fuzzy multiple attribute decision-making with incomplete weight information. Knowledge and Information Systems, 26, 337-349.

Xia, M.M., Xu, Z.S. (2011). Hesitant fuzzy information aggregation in decision making. International Journal of Approximate Reasoning, 52, 395-407.

Ye, J. (2010). Multicriteria fuzzy decision-making method using entropy weights-based correlation coefficients of interval-valued intuitionistic fuzzy sets. Applied Mathematical Modelling, 34, 3864-3870.

Ye, J. (2013). Multicriteria decision-making method using the correlation coefficient under single-valued neutrosophic environment. International Journal of General Systems, 42(4), 386-394. 
Ye, J. (2014a). Correlation coefficient of dual hesitant fuzzy sets and its application to multiple attribute decision making. Applied Mathematical Modelling, 38, 659-666.

Ye, J. (2014b). Single valued neutrosophic cross-entropy for multicriteria decision making problems. Applied Mathematical Modelling, 38, 1170-1175.

Ye, J. (2014c). Similarity measures between interval neutrosophic sets and their applications in multicriteria decision-making. Journal of Intelligent and Fuzzy Systems, 26, 165-172.

Ye, J. (2014d). A multicriteria decision-making method using aggregation operators for simplified neutrosophic sets. Journal of Intelligent and Fuzzy Systems, 26(5), 2459-2466.

J. Ye is graduated and received his MS degree in Automation and Robotics from the Technical University of Koszalin, Poland in 1997. From February 2012 to August 2012, he was a visiting scholar in the School of Engineering of Southern Polytechnic State University in USA. Now, he is a professor in the Department of Electrical and Information Engineering, Shaoxing University, China. He has more than 30 years of experience in teaching and research. His research interests include soft computing, fuzzy decision-making, pattern recognitions, fault diagnosis, robotics, and intelligent control. He has published more than one hundred papers in journals. He has written few books related to his research work. He has finished a few projects sponsored by government of China.

\section{Koreliacijos koeficientai intervalinėms neutrosofinėms atspariosioms aibėms ir jų taikymas daugiakriterinio sprendimų priẻmimo metode}

Jun YE

Šiame straipsnyje pasiūlyta intervalinių neutrosofinių atspariujų aibių (INAA) koncepcija ir apibrèžtos operacijos su INAA. Apibrèžiami INAA koreliacijos koeficientai ir tiriamas jų ryšys su panašumo matais. Be to, pasiūlytas daugiakriterinio sprendimų prièmimo metodas, paremtas koreliacijos koeficiento taikymu. Atsižvelgiant ị koreliacijos koeficientus tarp alternatyvų ir idealiosios alternatyvos, surandami alternatyvų rangai. Pateikiamas pasiūlyto metodo taikymo investiciniams sprendimams priimti pavyzdys. 